\title{
Anomalies in Ward Identities for Three-Point Functions Revisited
}

\author{
O.A. Battistel ${ }^{*}$ and O.L. Battistel ${ }^{* *}$ \\ *Dept. of Physics \\ Universidade Federal de Santa Maria, 5093, 97119-900, Santa Maria, RS, Brazil \\ **Exact Science Area \\ Centro Universitário Franciscano, Rua dos Andradas, 1614, 97010-032, Santa Maria, RS, Brazil
}

\begin{abstract}
A general calculational method is applied to investigate symmetry relations among divergent amplitudes in a free fermion model. A very traditional work on this subject is revisited. A systematic study of one, two and three point functions associated to scalar, pseudoscalar, vector and axial-vector densities is performed. The divergent content of the amplitudes are left in terms of five basic objects (external momentum independent). No specific assumptions about a regulator is adopted in the calculations. All ambiguities and symmetry violating terms are shown to be associated with only three combinations of the basic divergent objects. Our final results can be mapped in the corresponding Dimensional Regularization calculations (in cases where this technique could be applied) or in those of Gertsein and Jackiw which we will show in detail. The results emerging from our general approach allow us to extract, in a natural way, a set of reasonable conditions (e.g. crucial for QED consistency) that could lead us to obtain all Ward Identities satisfied. Consequently, we conclude that the traditional approach used to justify the famous triangular anomalies in perturbative calculations could be questionable. An alternative point of view, dismissed of ambiguities, which lead to a correct description of the associated phenomenology, is pointed out.
\end{abstract}

\section{INTRODUCTION}

Quantum Field Theory is nowadays our main tool for the investigation of the elementary particle interactions. This is also due to the fact that it allows us to study the consequences of symmetries assumed relevant, our fundamental working hypothesis. Since this is seldom possible, in an exact way, perturbative techniques become most relevant. Unfortunately, this type of solution is plagued with mathematical problems coming from divergent integrals. Therefore we are forced to have the recourse of auxiliary techniques based on regularization schemes in order to extract the physical content of calculated amplitudes. However, the regularization schemes invariably modify the amplitudes, as they are dictated by Feynman rules, and consequently provide only a particular interpretation for the mathematical indefinitions in the problem. Frequently, the final results become regularization-scheme dependent and may not reflect the full dynamical content of the underlying theory. In this context two of the main issues are the question of ambiguities associated with arbitrary momentum routing in loops and the symmetry relation violations [1]. Many of these fundamental questions have been adequately solved after the construction of Dimensional Regularization (DR) 22. There is however one important issue which, due to the mathematical limitation of the technique, could not be handled, namely, the pseudo-amplitudes. Perhaps this is the reason why even nowadays the treatment given to such amplitudes have the recourse of methods completely discarded in all cases where DR applies. We refer specifically to the problem of triangle anomalies, which has been discussed in the pioneer work of Gertsein and Jackiw [1]. They proposed that the ambiguous character of the amplitudes is associated to the existence of violations in symmetry relations. In their analysis, the surface terms associated to eventual shifts relating two different choices for the internal (arbitrary) momentum routing, play an important role. In spite of the relative success of this recipe there is a deeply frustrating aspect involved, namely, the assumption that the space-time homogeneity (translational invariance), one of the most basic symmetry implemented in the construction of quantum field theories, is lost in the perturbative calculations. In addition, the predictive power of such theories are affected because in cases where the amplitudes assume degrees of divergence higher than logarithmic this means that no unique answer can be obtained [3]. To decide by one of the infinite possible expressions for a physical amplitude we need some knowledge about the phenomenology or, in other words, to choose a value for each ambiguity involved. But there are some situations where a completely healthy amplitude cannot be accessed by choosing ambiguities. This is exactly the situation of the triangle anomalies 4 . This point of view, introduced by Gertsein and Jackiw [1], before the advent of DR technique, remains as the accepted one even in modern quantum field theory text books [5], [6]: the violation of symmetry relations (anomalies) in perturbative calculations is associated to the divergent (ambiguous) character of the amplitudes. This is a completely discarded picture in all other situations involving divergences in perturbative evaluations of physical amplitudes. Anomalies, in other hand, are shown to emerge even in theories dismissed of divergences [6], [7] and predicted by several arguments in QFT [6], [8]. It seems to have no doubt about their existence. So, if there is a fundamental quantum phenomenon of the nature associated to anomalies [9], it must be present even in exact solutions 
of the theory (dismissed of infinities and ambiguities). Therefore it is reasonable to believe that it must be possible to establish their existence without having to appeal to ambiguities, even in perturbative calculations where divergent amplitudes are involved.

Ambiguities and symmetry violations in perturbative calculations, in other hand, remain as unsolved questions even for many others than anomaly problems. Discussions involving these aspects can be copiously found in the recent literature in different contexts but playing a crucial role in the job of the QFT theoretical methods description of a particular phenomenology. In the case of effective non-renormalizable models, like NJL model [10], ambiguities and symmetry violations are deeply related with the own predictive power of such models [11]. Investigations on this subject have pointed out that usual procedures like DR, sharp cut off and Pauli-Villars cannot offer acceptable results [12]. More recently the role of ambiguities have emerged in the context of Lorentz and CPT violations in an extended version of Standard Model [13]. Perturbative evaluations of the radiative contributions to a Lorentz and CPT breaking coupling of the pure photon sector of an extended QED, of the Chern-Simmons form, are verified to be regularization-scheme dependent and plagued by the ambiguities. In addition many aspects of the discussions about the controversy involving the Chern-Simmons shift are closely related to that of $A V V$ anomaly once the mathematical structure of the (divergent) contributions are completely similar 14. Explicitly evaluation of the radiatively induced Chern-Simmons term using DR [15, Differential Regularization [16] and surface term evaluation [14], among others [17], cannot lead us to a definite value, i.e., only an ambiguous value can be extracted using traditional techniques.

Ambiguities, symmetry relation violations and regularization-scheme dependence are also present in the aim of the investigations about the implications of the fermionic tensor densities for low energy hadronic observable, a completely new physics [18].

All the above cited problems, and certainly others omitted here, constitute strong indications that additional investigations on the subject of ambiguities, symmetry relation violations and regularization-scheme dependence in perturbative calculations are in order. In light of these statements we have devoted this work to the investigation of the ambiguities and symmetry relations in a free (Dirac) fermion model. This is not more than an amplified revisiting to this question in a closely related way with the study that was done by Gertsein and Jackiw in ref. [1], a historical paper on this subject. Our intention is to use a general calculational method to manipulate and calculate the divergent integrals in such a way that all amplitudes in different theories and models are handled by the same prescription and connections with conventional techniques could be established. A such alternative strategy to manipulate and calculate divergences in perturbative calculations have been proposed [19] and successfully applied in the treatment of the ambiguities and symmetry relation violations in the context of the gauged NJL Model 20] and to investigate the possibility of a radiatively induced Chern-Simmons shift in an extended version of QED [21]. This method so far has proved to be an adequate tool to treat all questions related with divergences from the renormalization of standard theories like QED [22], calculation of renormalization group coefficients [23] and, for calculations in effective nonrenormalizable theories 19. Having this in mind we turn attention in the present work perhaps to the historically most relevant question that involves the association between the violation of symmetry relations and ambiguities. The advantage of the method adopted here is that it allows an immediate connection with other current approaches. In particular, we can use the consistent results obtained by DR, where it applies, as element of the analysis for the search of an universal interpretation for the divergences in physical amplitudes. From the theoretical point of view an adequate description of the anomaly phenomenon, and any other in the perturbative calculations, is only achieved if for identical mathematical objects an identical value is attributed in all situations where it occurs. In this context, it is most desirable to obtain the anomalies in a natural way within a context which treats all anomalous and non-anomalous amplitudes according to the same scheme. In this direction the first step is the verification if the current traditional approach used to justify the triangle anomalies produces results in a consistent way with those of Dimensional Regularization where both procedures could be applied. One must check whether the current way as followed in ref. [1] for calculating e.g. the famous axial anomaly remains appropriate when used to investigate other symmetry relations. In other words, anomalies must also emerge in a treatment designed to consistently handle all other amplitudes, i.e., where ambiguities are not necessarily present, in spite of the divergent character.

After detailed results have been obtained, in the point of view of our general calculational method, we will show that the results can be mapped separately in those corresponding of Dimensional Regularization and to those of surface's terms analysis of ref. [1], but these two prescriptions are conflicting, i.e., cannot be simultaneously mapped by an universal interpretation. An important aspect involved in this investigation is that when we demand a complete mapping of our results (in 4-D) with those of DR we obtain a set of conditions which are not compatible with any symmetry violations, even for the anomalous cases. This is an indication that the procedure adopted in ref. [1] to establish the triangle anomaly cannot be considered as a consistent one. We conclude that this is due to the fact that only two-point functions are explicitly calculated in the traditional procedure and the violation of symmetry relations in the anomalous amplitudes are intrinsic properties of the three-point functions involved. A consistent calculation of the $A V V$ and $A A A$ amplitudes reveals that the violations of the Ward Identities characterizing the anomaly phenomenon emerges, in a natural way, with the expected value, without having to admit ambiguities and 
within an interpretation which is universal in a sense that consistent results are obtained for all amplitudes in the same point of view [19].

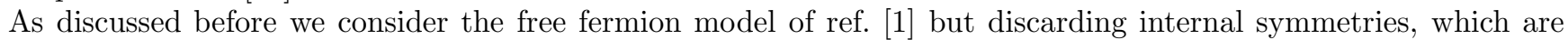
irrelevant for our purposes. In section II we define the model, notation and relevant Ward Identities. In section III we briefly establish the operational strategy for the manipulation and calculation of divergent amplitudes. In section IV a study of ambiguities is presented in one and two-point functions calculated explicitly with arbitrary momentum routing. We also explicitate the ambiguous terms of the three-point functions. In section V we reproduce (from our results) those of ref. [1]. In section VI we discuss Ward Identities. The final remarks are contained in section VII.

\section{DEFINITIONS, NOTATION AND CURRENT ALGEBRA RESULTS FOR THE MODEL}

We start introducing the notation to be used and defining the quantities we will be concerned with for the rest of the work. We closely follow ref. [1] with which we compare our results.

Let us consider a spin $1 / 2$, mass $m$ free fermion model. There will be therefore a massive field which obeys Dirac's equation and with which we can construct currents $j_{i}(x)$ defined by

$$
j_{i}(x)=\bar{\psi}(x) \Gamma_{i} \psi(x)
$$

where $\Gamma_{i}$ are the Dirac's matrices responsible for the transformation properties of the currents:

$$
\Gamma_{i}=\left[\hat{1} ; \gamma_{5} ; \gamma_{\mu} ; i \gamma_{\mu} \gamma_{5}\right]
$$

characterizing the scalar $S(x)$, pseudo-scalar $P(x)$, vector $V_{\mu}(x)$ and axial-vector $A_{\mu}(x)$ densities, respectively. Tensor densities will be considered elsewhere [28]. An important property in this model is the value of the four divergences

$$
\left\{\begin{array}{c}
\partial_{\mu} V_{\mu}(x)=0 \\
\partial_{\mu} A_{\mu}(x)=2 m P(x),
\end{array}\right.
$$

and their commutation relations at equal times [1]. With the above definitions and the fermionic propagator

$$
i S_{F}(p)=\frac{i}{\not p-m}
$$

it is possible to construct n-point functions, which we define in the same way as in ref. [1] as follows

- One-point functions:

$$
T^{i}\left(k_{1}, m\right)=\int \frac{d^{4} k}{(2 \pi)^{4}} \operatorname{Tr}\left\{\Gamma_{i} \frac{1}{\left[\left(\not k+\not k_{1}\right)-m\right]}\right\}
$$

- Two-point functions:

$$
T^{i j}\left(k_{1}, m ; k_{2}, m\right)=\int \frac{d^{4} k}{(2 \pi)^{4}} \operatorname{Tr}\left\{\Gamma_{i} \frac{1}{\left[\left(\not k+\not k_{1}\right)-m\right]} \Gamma_{j} \frac{1}{\left[\left(\not k+\not k_{2}\right)-m\right]}\right\},
$$

- Three-point functions:

$$
\begin{aligned}
& T^{l i j}\left(k_{1}, m ; k_{2}, m ; k_{3}, m\right)= \\
& \quad \int \frac{d^{4} k}{(2 \pi)^{4}} \operatorname{Tr}\left\{\Gamma_{l} \frac{1}{\left[\left(\not k+\not k_{3}\right)-m\right]} \Gamma_{i} \frac{1}{\left[\left(\not k+\not k_{1}\right)-m\right]} \Gamma_{j} \frac{1}{\left[\left(\not k+\not k_{2}\right)-m\right]}\right\},
\end{aligned}
$$

and so on. Here the $k_{1}, k_{2}$ and $k_{3}$ represent the arbitrary choices for the internal momenta of the loop. Energy momentum conservation only requires these quantities must be related with the external momenta, e.g., in the threepoint functions we have: $k_{3}-k_{1}=p, k_{1}-k_{2}=p^{\prime}$ and $k_{3}-k_{2}=q$.

In our notation the vertex to the left is assumed to be connected with the "initial state" with external momentum $q$ and the vertex operator $\Gamma_{l}$. The other two vertices correspond to "final states" with external momenta $p$ and $p^{\prime}$ and the vertex operators $\Gamma_{i}$ e $\Gamma_{j}$, respectively. Besides the upper indices are associated, in the order they appear, with 
the respective Lorentz indices in the same order, whenever is the case. As an example, if $\Gamma_{l}=\gamma_{\lambda}$, $\Gamma_{i}=i \gamma_{\mu} \gamma_{5}$ and $\gamma_{j}=\gamma_{5}$ we have

$$
T_{\lambda \mu}^{V A P}\left(k_{1}, m ; k_{2}, m ; k_{3}, m\right)
$$

This notation emphasizes the masses and momenta carried by the internal lines and characterizes each diagram completely. In particular this means that in the case we have one particle in the initial state and two on the final one, symmetrization in the final states will be required. For example for the process $S \rightarrow V V$ we define the corresponding amplitude as

$$
T_{\mu \nu}^{S \rightarrow V V}=T_{\mu \nu}^{S V V}\left(k_{1}, m ; k_{2}, m ; k_{3}, m\right)+T_{\nu \mu}^{S V V}\left(l_{1}, m ; l_{2}, m ; l_{3}, m\right)
$$

The first term represents the direct channel and the second the crossed channel. In the second term $l_{1}, l_{2}$ e $l_{3}$ are the arbitrary choices for the corresponding internal momenta.

There are integral representations for the functions defined above with the Fourier transform of the currents

$$
<j_{1}(q) j_{2}(-q)>\equiv \int e^{-i p x} d^{4} x<0 \mid T\left(j_{1}(x) j_{2}(0) \mid 0>\right.
$$

in the case of two-point functions, and

$$
<j_{1}(p) j_{2}\left(p^{\prime}\right) j_{3}(q)>\equiv \int e^{-i p x} e^{-i p^{\prime} y} d^{4} x d^{4} y<0\left|T\left(j_{1}(x) j_{2}(y) j_{3}(0)\right)\right| 0>
$$

for the three-point functions. With these elements and the standard methods of the current algebra [1], [4], one can establish relations among the n-point functions, i.e., Ward Identities. For the simple model in question such symmetry relations are reduced to the conservation of the vector current and the well-known proportionality of the divergence of the axial current and the pseudoscalar one. It is important to remark that such relations are exact and should be satisfied in spite of the divergent character of up to $n$-point functions.

This brief discussion summarizes the dilemma one has to face when calculating divergent amplitudes: to maintain their properties after the calculation. It is our purpose to investigate under which conditions it is possible to evaluate these amplitudes and to get consistent results in what concerns ambiguities and symmetry relations.

The Ward Identities that we should verify are the following

- One-point function

$$
T_{\mu}^{V}\left(k_{1}, m\right)=0
$$

- Two-point functions

$$
\begin{gathered}
\left(k_{1}-k_{2}\right)_{\mu} T_{\mu}^{V S}\left(k_{1}, m ; k_{2}, m\right)=0 \\
\left(k_{1}-k_{2}\right)_{\mu} T_{\mu \nu}^{V V}\left(k_{1}, m ; k_{2}, m\right)=0 \\
\left(k_{1}-k_{2}\right)_{\nu} T_{\mu \nu}^{V V}\left(k_{1}, m ; k_{2}, m\right)=0 \\
\left(k_{1}-k_{2}\right)_{\mu} T_{\mu}^{A P}\left(k_{1}, m ; k_{2}, m\right)=-2 m i T^{P P}\left(k_{1}, m ; k_{2}, m\right) \\
\left(k_{1}-k_{2}\right)_{\mu} T_{\mu \nu}^{A V}\left(k_{1}, m ; k_{2}, m^{2}\right)=-2 m i T_{\nu}^{P V}\left(k_{1}, m ; k_{2}, m\right)-i T^{S}\left(k_{1}, m\right)-i T^{S}\left(k_{2}, m\right) \\
\left(k_{1}-k_{2}\right)_{\nu} T_{\mu \nu}^{A V}\left(k_{1}, m ; k_{2}, m\right)=0 \\
\left(k_{1}-k_{\mu \nu}^{A A}\left(k_{1}, m ; k_{2}, m\right)=-2 m i T_{\nu}^{P A}\left(k_{1}, m ; k_{2}, m\right)\right. \\
\left.k_{1}-k_{2}\right)_{\mu} T_{\mu \nu}^{A A}\left(k_{1}, m ; k_{2}, m\right)=(2 m)^{2} T^{P P}\left(k_{1}, m ; k_{2}, m\right)+2 m T^{S}\left(k_{1}, m\right)+2 m T^{S}\left(k_{2}, m\right)
\end{gathered}
$$


- Three-point functions

$$
\begin{aligned}
& q_{\lambda} T_{\lambda}^{V \rightarrow S S}=0 \\
& q_{\lambda} T_{\lambda}^{V \rightarrow P P}=0 \\
& q_{\lambda} T_{\lambda}^{A \rightarrow S P}=-2 m i T^{P \rightarrow S P} \\
& p_{\nu}^{\prime} T_{\mu \nu}^{S \rightarrow V V}=0 \\
& p_{\mu} T_{\mu \nu}^{S \rightarrow V V}=0 \\
& p_{\mu} T_{\mu \nu}^{S \rightarrow A A}=2 m i T_{\nu}^{S \rightarrow P A} \\
& p_{\nu}^{\prime} T_{\mu \nu}^{S \rightarrow A A}=2 m i T_{\mu}^{S \rightarrow A P} \\
& p_{\mu} T_{\mu \nu}^{P \rightarrow A V}=2 m i T_{\nu}^{P \rightarrow P V} \\
& p_{\nu}^{\prime} T_{\mu \nu}^{P \rightarrow A V}=0 \\
& p_{\mu} T_{\mu \nu}^{P \rightarrow V V}=0 \\
& p_{\nu}^{\prime} T_{\mu \nu}^{P \rightarrow V V}=0 \\
& p_{\mu} T_{\mu \nu}^{S \rightarrow A V}=2 m i T_{\nu}^{S \rightarrow P V} \\
& p_{\nu}^{\prime} T_{\mu \nu}^{S \rightarrow A V}=0 \\
& p_{\mu} T_{\mu \nu}^{P \rightarrow A A}=2 m i T_{\nu}^{P \rightarrow P A} \\
& p_{\nu}^{\prime} T_{\mu \nu}^{P \rightarrow A A}=2 m i T_{\mu}^{P \rightarrow A P} \\
& q_{\lambda} T_{\lambda \mu \nu}^{A \rightarrow V V}=-2 m i T_{\mu \nu}^{P \rightarrow V V} \\
& p_{\mu} T_{\lambda \mu \nu}^{A \rightarrow V V}=0 \\
& p_{\nu}^{\prime} T_{\lambda \mu \nu}^{A \rightarrow V V}=0 \\
& q_{\lambda} T_{\lambda \mu \nu}^{A \rightarrow A A}=-2 m i T_{\mu \nu}^{P \rightarrow A A} \\
& p_{\mu} T_{\lambda \mu \nu}^{A \rightarrow A A}=2 m i T_{\lambda \nu}^{A \rightarrow P A} \\
& p_{\nu}^{\prime} T_{\lambda \mu \nu}^{A \rightarrow A A}=-2 m i T_{\lambda \mu}^{A \rightarrow P A} \\
& q_{\lambda} T_{\lambda \mu \nu}^{V \rightarrow V V}=0 \\
& p_{\mu} T_{\lambda \mu \nu}^{V \rightarrow V V}=0 \\
& p_{\nu}^{\prime} T_{\lambda \mu \nu}^{V \rightarrow V V}=0 \\
& q_{\lambda} T_{\lambda \mu \nu}^{V \rightarrow A A}=0 \\
& p_{\mu} T_{\lambda \mu \nu}^{V \rightarrow A A}=2 m i T_{\lambda \nu}^{V \rightarrow P A} \\
& p_{\nu}^{\prime} T_{\lambda \mu \nu}^{V \rightarrow A A}=2 m i T_{\lambda \mu}^{V \rightarrow A P}
\end{aligned}
$$




\section{CALCULATIONAL STRATEGY TO MANIPULATE DIVERGENT INTEGRALS}

For the verification of the above cited Ward Identities it becomes necessary some explicitly evaluation of the divergent amplitudes. In face of the well-known mathematical indefinitions involved we need to adopt some strategy to handle this problem. The usual procedure is the adoption of a regularization scheme or any equivalent philosophy. For the following calculations we will adopt an alternative strategy. Rather than the specification of some regularization, to justify all the manipulations, we will assume the presence of a regulating distribution only in an implicitly way. Schematically:

$$
\int \frac{d^{4} k}{(2 \pi)^{4}} f(k) \rightarrow \int \frac{d^{4} k}{(2 \pi)^{4}} f(k)\left\{\lim _{\Lambda_{i}^{2} \rightarrow \infty} G_{\Lambda_{i}}\left(k^{2}, \Lambda_{i}^{2}\right)\right\}=\int_{\Lambda} \frac{d^{4} k}{(2 \pi)^{4}} f(k) .
$$

Here $\Lambda_{i}^{\prime} s$ are parameters of the generic distribution $G\left(\Lambda_{i}^{2}, k^{2}\right)$. In addition to the obvious finiteness of the modified integral, we require two very general properties of $G\left(\Lambda_{i}^{2}, k^{2}\right)$; it should be even in the integrating momentum $k$, due to Lorentz invariance maintenance, and a well-defined connection limit should exists, i.e., $\lim _{\Lambda_{i}^{2} \rightarrow \infty} G_{\Lambda_{i}}\left(k^{2}, \Lambda_{i}^{2}\right)=1$. The first property imply that all odd integrands vanishes. The second one guarantees, in particular, that the value of finite integrals in the amplitudes will not be modified. In the next step we manipulate the integrand of the divergent integrals by using identities to generate a mathematical expression where all the divergences are contained in external momenta independent structures. This goal can be achieved by the use of an adequate identity like:

$$
\frac{1}{\left[\left(k+k_{i}\right)^{2}-m^{2}\right]}=\frac{1}{\left(k^{2}-m^{2}\right)}+\sum_{j=1}^{N} \frac{(-1)^{j}\left(k_{i}^{2}+2 k_{i} \cdot k\right)^{j}}{\left(k^{2}-m^{2}\right)^{j+1}}+\frac{(-1)^{N+1}\left(k_{i}^{2}+2 k_{i} \cdot k\right)^{N+1}}{\left(k^{2}-m^{2}\right)^{N+1}\left[\left(k+k_{i}\right)^{2}-m^{2}\right]}
$$

where $k_{i}$ is (in principle) an arbitrary momentum. The minor value of $N$ should be the one that leads the last term in above expression to a finite integral, and then, in face of the connection limit requirement, the corresponding integration can be performed without restriction. The divergent structures obtained this way have no additional assumptions and (in the discussed problem) they are written as a combination of five objects, namely:

$$
\begin{aligned}
\bullet \square_{\alpha \beta \mu \nu}= & \int_{\Lambda} \frac{d^{4} k}{(2 \pi)^{4}} \frac{24 k_{\mu} k_{\nu} k_{\alpha} k_{\beta}}{\left(k^{2}-m^{2}\right)^{4}}-g_{\alpha \beta} \int_{\Lambda} \frac{d^{4} k}{(2 \pi)^{4}} \frac{4 k_{\mu} k_{\nu}}{\left(k^{2}-m^{2}\right)^{3}} \\
& -g_{\alpha \nu} \int_{\Lambda} \frac{d^{4} k}{(2 \pi)^{4}} \frac{4 k_{\beta} k_{\mu}}{\left(k^{2}-m^{2}\right)^{3}}-g_{\alpha \mu} \int_{\Lambda} \frac{d^{4} k}{(2 \pi)^{4}} \frac{4 k_{\beta} k_{\nu}}{\left(k^{2}-m^{2}\right)^{3}} \\
\bullet \Delta_{\mu \nu}= & \int_{\Lambda} \frac{d^{4} k}{(2 \pi)^{4}} \frac{4 k_{\mu} k_{\nu}}{\left(k^{2}-m^{2}\right)^{3}}-\int_{\Lambda} \frac{d^{4} k}{(2 \pi)^{4}} \frac{g_{\mu \nu}}{\left(k^{2}-m^{2}\right)^{2}} \\
\bullet \nabla_{\mu \nu}= & \int_{\Lambda} \frac{d^{4} k}{(2 \pi)^{4}} \frac{2 k_{\nu} k_{\mu}}{\left(k^{2}-m^{2}\right)^{2}}-\int_{\Lambda} \frac{d^{4} k}{(2 \pi)^{4}} \frac{g_{\mu \nu}}{\left(k^{2}-m^{2}\right)} \\
\bullet I_{l o g}\left(m^{2}\right)= & \int_{\Lambda} \frac{d^{4} k}{(2 \pi)^{4}} \frac{1}{\left(k^{2}-m^{2}\right)^{2}} \\
\bullet I_{\text {quad }}\left(m^{2}\right)= & \int_{\Lambda} \frac{d^{4} k}{(2 \pi)^{4}} \frac{1}{\left(k^{2}-m^{2}\right)} .
\end{aligned}
$$

With this strategy, it became possible to map the final expressions obtained by us into the corresponding results of other techniques, once all the steps are perfectly valid with reasonable regularization prescriptions, including those of DR. All we need is to evaluate the divergent structures obtained with the specific philosophy which we will make contact. In addition we call attention to the fact that no shifts will be used on the general routing assumed for all amplitudes. Consequently it will be possible to make contact with those results corresponding to explicit evaluation of surfaces terms in eventual shifts performed in the integrating momentum of the loop integrals [1]. This very general character of the adopted strategy will become the most important ingredient for the analysis we want to do and to support our conclusions. Another important aspect of the procedure is that a definite value for each divergent integral involved is attributed. This value is used always that the integrals are present in a physical amplitude in all theories and models, providing, in this way, an universal point of view for the problem. No expansions, limits or not totally controlled procedures are included. All the manipulations and calculations we need in treatment of divergences in QFT is performed without the explicit calculation of a divergent integral as become clear in what follows. 


\section{AMBIGUITIES}

The integral representation, eq.(5), for one-point functions indicates, by power counting, that there is a cubic degree of divergence. Also, eq.(6) reveals that the two-point functions are quadratically divergent and eq.(7) indicates a linearly one for the three-point functions. In the point of view adopted in ref. [1], all these quantities are, in principle, ambiguous quantities. The reason for this resides on the fact that the energy-momentum conservation relations do not uniquely specify the internal momenta in the loop. It is possible to make different choices for the internal momentum label. Such choices could only be equivalent if shifts in the integrating variable were allowed, which is not the case when the degree of divergence is higher than logarithmic [5], [6]. In what follows we will explicitly evaluate one and two-point functions in detail from the point of view of our general calculational method. For the three-point functions only the potentially ambiguous terms are explicitly showed as required for the analysis we want to do, closely related to that of ref. [1]. All calculations will be made adopting general routing for the internal momenta in such a way all possible choices are automatically included. After that all desired results have been obtained and a mapping to those of ref. [1] produced, we will analyze all possible ambiguities. An amazing simple structure for these ambiguities will emerge so that immediate and transparent conclusions can be extracted.

\section{A. One-Point Functions}

We start by considering the one-point vector amplitude $T_{\mu}^{V}$. According to the standard definition this function contains one fermionic propagator and the vertex operator $\Gamma_{i}=\gamma_{\mu}$. It is defined, following our notation, as

$$
T_{\mu}^{V}=\int \frac{d^{4} k}{(2 \pi)^{4}} \operatorname{Tr}\left\{\gamma_{\mu} \frac{1}{\left(k+\not k_{1}\right)-m}\right\}
$$

and, after the trace is taken, we get

$$
T_{\mu}^{V}=4\left\{\int \frac{d^{4} k}{(2 \pi)^{4}} \frac{k_{\mu}}{\left(k+k_{1}\right)^{2}-m^{2}}+k_{1 \mu} \int \frac{d^{4} k}{(2 \pi)^{4}} \frac{1}{\left(k+k_{1}\right)^{2}-m^{2}}\right\} .
$$

From the above equation we see that we get two divergent integrals, one of cubic and the other of quadratic degree of divergences. Following our strategy we admit the presence of an implicit regulator, as discussed before. In order to indicate its presence we use the subscript $\Lambda$ in the integral and proceed to the necessary manipulations of the integrand in such a way that the dependence on $k_{1}$ momentum is present only in finite integrals in which case the connection limit is invocated. This goal can be achieved by the use of the identity eq.(49) choosing $N$ not minor than three for the cubic divergent integral above. Proceeding on this way we get:

$$
\begin{aligned}
\int_{\Lambda} \frac{d^{4} k}{(2 \pi)^{4}} \frac{k_{\mu}}{\left(k+k_{1}\right)^{2}-m^{2}}= & -k_{1 \nu}\left\{\int_{\Lambda} \frac{d^{4} k}{(2 \pi)^{4}} \frac{2 k_{\mu} k_{\nu}}{\left(k^{2}-m^{2}\right)^{2}}\right\} \\
& +k_{1 \nu} k_{1 \alpha} k_{1 \beta}\left\{\int_{\Lambda} \frac{d^{4} k}{(2 \pi)^{4}} \frac{4 g_{\alpha \beta} k_{\mu} k_{\nu}}{\left(k^{2}-m^{2}\right)^{3}}-\int_{\Lambda} \frac{d^{4} k}{(2 \pi)^{4}} \frac{8 k_{\alpha} k_{\beta} k_{\mu} k_{\nu}}{\left(k^{2}-m^{2}\right)^{4}}\right\} \\
& -\left\{\int \frac{d^{4} k}{(2 \pi)^{4}} \frac{6 k_{1}^{4} k_{1 \nu} k_{\mu} k_{\nu}}{\left(k^{2}-m^{2}\right)^{4}}-\int \frac{d^{4} k}{(2 \pi)^{4}} \frac{\left(k_{1}^{2}+2 k_{1} \cdot k\right)^{4} k_{\mu}}{\left(k^{2}-m^{2}\right)^{4}\left[\left(k+k_{1}\right)^{2}-m^{2}\right]}\right\} .
\end{aligned}
$$

Note the absence of the odd integrals in consequence of the even character of our implicit regulator and that we have removed the subscript $\Lambda$ on the two last integrals in consequence of the connection limit requirement. The finite integrals so obtained can be solved by standard techniques without any restriction. The result is an exact cancellation between them.

For the quadratic divergent integral in eq.(56) we apply the same recipe. Choosing in the eq.(49) the value $N=2$ we get

$$
\begin{aligned}
\int_{\Lambda} \frac{d^{4} k}{(2 \pi)^{4}} \frac{1}{\left(k+k_{1}\right)^{2}-m^{2}}= & \int_{\Lambda} \frac{d^{4} k}{(2 \pi)^{4}} \frac{1}{\left(k^{2}-m^{2}\right)} \\
& +k_{1 \mu} k_{1 \nu}\left\{\int_{\Lambda} \frac{d^{4} k}{(2 \pi)^{4}} \frac{4 k_{\mu} k_{\nu}}{\left(k^{2}-m^{2}\right)^{3}}-\int_{\Lambda} \frac{d^{4} k}{(2 \pi)^{4}} \frac{g_{\mu \nu}}{\left(k^{2}-m^{2}\right)^{2}}\right\} \\
& +\left\{\int \frac{d^{4} k}{(2 \pi)^{4}} \frac{k_{1}^{4}}{\left(k^{2}-m^{2}\right)^{3}}-\int \frac{d^{4} k}{(2 \pi)^{4}} \frac{\left(k_{1}^{2}+2 k_{1} \cdot k\right)^{3}}{\left(k^{2}-m^{2}\right)^{3}\left[\left(k+k_{1}\right)^{2}-m^{2}\right]}\right\}
\end{aligned}
$$


where, again, the last two integrals cancel out after that the integration have been performed. Then, collecting the results of eq.(57) and eq.(58) without any modification we get for the $T_{\mu}^{V}$ one-point function

$$
T_{\mu}^{V}=4\left\{-k_{1 \beta} \nabla_{\beta \mu}-\frac{k_{1 \beta} k_{1 \alpha} k_{1 \nu}}{3} \square_{\alpha \beta \mu \nu}+\frac{k_{1}^{2} k_{1 \nu}}{3} \triangle_{\nu \mu}+\frac{2}{3} k_{1 \mu} k_{1 \alpha} k_{1 \beta} \triangle_{\alpha \beta}\right\},
$$

where we have introduced a set of differences between divergent integrals with the same degree of divergence, defined in eqs.(50)-(52). As announced on the preceding comments we will analyze the results only after all calculations have been performed. For while we only call the attention for the very general character of the assumptions we have made to produce the result eq.(59). No specific assumptions about a regulator are used and no shift, limits, expansions and so on involving the divergent parts are assumed. This is due to that, in fact, no calculations of divergent integrals have been made.

Let us now treat the remaining one-point functions within the same calculational scheme. Taking the scalar one, defined by

$$
T^{S}=\int \frac{d^{4} k}{(2 \pi)^{4}} \operatorname{Tr}\left\{\hat{1} \frac{1}{\left(\not k+\not k_{1}\right)-m}\right\}
$$

we get, after Dirac's trace,

$$
T^{S}=4 m \int \frac{d^{4} k}{(2 \pi)^{4}} \frac{1}{\left(k+k_{1}\right)^{2}-m^{2}} .
$$

The quadratically divergent integral in the above expression has already been discussed in the previous calculation. In consequence the one-point function $T^{S}$ can be written as

$$
T^{S}=4 m\left\{I_{\text {quad }}\left(m^{2}\right)+k_{1 \beta} k_{1 \alpha} \triangle_{\beta \alpha}\right\} .
$$

In the above result we verify that the amplitude $T^{S}$ is expressed in terms of two divergent objects, $I_{\text {quad }}\left(m^{2}\right)$ and $\triangle_{\beta \alpha}$, the latter again related to the arbitrary momentum label $k_{1}$. For the moment we only comment that the two calculated amplitudes are potentially ambiguous due to the presence of such terms in the final expression.

It is a simple matter to check that the remaining one-point functions $T_{\mu}^{A}$ and $T^{P}$ vanish identically due to the trace's properties of the Dirac's matrices involved.

\section{B. Two-Point Functions}

Let us calculate the two-point functions within the same scheme. We start by considering the simplest one of such set of functions, namely, the scalar-scalar, defined by

$$
T^{S S}=\int \frac{d^{4} k}{(2 \pi)^{4}} \operatorname{Tr}\left\{\hat{1} \frac{1}{\left(\not k+\not k_{1}\right)-m} \hat{1} \frac{1}{\left(\not k+\not k_{2}\right)-m}\right\}
$$

where $k_{1}$ and $k_{2}$ stand for the arbitrary choices for the internal momenta. Only the difference between them is a physical quantity, the external momentum, consequently other combinations than the difference in the result for the two-point functions will systematically locate ambiguities, wherever the case may be. After taking Dirac traces and some standard algebraic manipulations we get

$$
\begin{aligned}
T^{S S}= & 2\left\{\int \frac{d^{4} k}{(2 \pi)^{4}} \frac{1}{\left(k+k_{1}\right)^{2}-m^{2}}+\int \frac{d^{4} k}{(2 \pi)^{4}} \frac{1}{\left(k+k_{2}\right)^{2}-m^{2}}\right. \\
& \left.+\left[4 m^{2}-\left(k_{1}-k_{2}\right)^{2}\right] \int \frac{d^{4} k}{(2 \pi)^{4}} \frac{1}{\left[\left(k+k_{1}\right)^{2}-m^{2}\right]\left[\left(k+k_{2}\right)^{2}-m^{2}\right]}\right\},
\end{aligned}
$$

where we have identified the quadratically divergent integrals which already appeared in previous calculations. As for the second, logarithmically divergent, we apply the appropriate manipulations to cast it into the form: 


$$
\begin{aligned}
\int_{\Lambda} \frac{d^{4} k}{(2 \pi)^{4}} \frac{1}{\left[\left(k+k_{1}\right)^{2}-m^{2}\right]\left[\left(k+k_{2}\right)^{2}-m^{2}\right]}= & \int_{\Lambda} \frac{d^{4} k}{(2 \pi)^{4}} \frac{1}{\left(k^{2}-m^{2}\right)^{2}} \\
& -\int \frac{d^{4} k}{(2 \pi)^{4}} \frac{\left(k_{1}^{2}+2 k_{1} \cdot k\right)}{\left[\left(k^{2}-m^{2}\right)^{2}\right]\left[\left(k+k_{1}\right)^{2}-m^{2}\right]} \\
& -\int \frac{d^{4} k}{(2 \pi)^{4}} \frac{\left(k_{2}^{2}+2 k_{2} \cdot k\right)}{\left[\left(k^{2}-m^{2}\right)^{2}\right]\left[\left(k+k_{2}\right)^{2}-m^{2}\right]} \\
& +\int \frac{d^{4} k}{(2 \pi)^{4}} \frac{\left(k_{1}^{2}+2 k_{1} \cdot k\right)\left(k_{2}^{2}+2 k_{2} \cdot k\right)}{\left[\left(k^{2}-m^{2}\right)^{2}\right]\left[\left(k+k_{1}\right)^{2}-m^{2}\right]\left[\left(k+k_{2}\right)^{2}-m^{2}\right]},
\end{aligned}
$$

where we chosen, in the eq.(49), $N=1$ for the two propagators involved which is very convenient, instead not unique, since it maintains the symmetry in $k_{1}$ and $k_{2}$. The divergent content of this amplitude is contained in the basic divergent object $I_{l o g}$. The remaining integrals are finite and yield

$$
\int \frac{d^{4} k}{(2 \pi)^{4}} \frac{\left(k_{1}^{2}+2 k_{1} \cdot k\right)}{\left[\left(k^{2}-m^{2}\right)^{2}\right]\left[\left(k+k_{1}\right)^{2}-m^{2}\right]}=\left(\frac{i}{(4 \pi)^{2}}\right)\left[Z_{0}\left(m^{2}, m^{2}, k_{1}^{2} ; m^{2}\right)\right],
$$

and

$$
\begin{aligned}
& \int \frac{d^{4} k}{(2 \pi)^{4}} \frac{\left(k_{1}^{2}+2 k_{1} \cdot k\right)\left(k_{2}^{2}+2 k_{2} \cdot k\right)}{\left[\left(k^{2}-m^{2}\right)^{2}\right]\left[\left(k+k_{1}\right)^{2}-m^{2}\right]\left[\left(k+k_{2}\right)^{2}-m^{2}\right]}= \\
& \left(\frac{i}{(4 \pi)^{2}}\right)\left[Z_{0}\left(m^{2}, m^{2}, k_{1}^{2} ; m^{2}\right)+Z_{0}\left(m^{2}, m^{2}, k_{2}^{2} ; m^{2}\right)-Z_{0}\left(m^{2}, m^{2},\left(k_{1}-k_{2}\right)^{2} ; m^{2}\right)\right],
\end{aligned}
$$

where we leave the integration in the last of Feynman parameters through the introduction of the structure functions for one loop integrals defined as [19]

$$
Z_{k}\left(\lambda_{1}^{2}, \lambda_{2}^{2}, q^{2} ; \lambda^{2}\right)=\int_{0}^{1} d z z^{k} \ln \left(\frac{q^{2} z(1-z)+\left(\lambda_{1}^{2}-\lambda_{2}^{2}\right) z-\lambda_{1}^{2}}{\left(-\lambda^{2}\right)}\right),
$$

which have been proven very useful in the systematization of this type of calculations. Explicit expressions for $Z_{k}$ functions can be easily obtained but are not relevant on this investigations. Collecting the results, the logarithmically divergent integral can be written as

$$
\int \frac{d^{4} k}{(2 \pi)^{4}} \frac{1}{\left[\left(k+k_{1}\right)^{2}-m^{2}\right]\left[\left(k+k_{2}\right)^{2}-m^{2}\right]}=I_{l o g}\left(m^{2}\right)-\left(\frac{i}{(4 \pi)^{2}}\right) Z_{0}\left(m^{2}, m^{2},\left(k_{1}-k_{2}\right)^{2} ; m^{2}\right)
$$

and, consequently, the $T^{S S}$ amplitude is given by

$$
\begin{aligned}
T^{S S}= & 4\left\{I_{\text {quad }}\left(m^{2}\right)+\frac{4 m^{2}-\left(k_{1}-k_{2}\right)^{2}}{2}\left[I_{\text {log }}\left(m^{2}\right)\right]\right. \\
& \left.-\frac{4 m^{2}-\left(k_{1}-k_{2}\right)^{2}}{2}\left(\frac{i}{(4 \pi)^{2}}\right) Z_{0}\left(m^{2}, m^{2},\left(k_{1}-k_{2}\right)^{2} ; m^{2}\right)\right\} \\
& +\left[\left(k_{1}-k_{2}\right)_{\alpha}\left(k_{1}-k_{2}\right)_{\beta}\right] \triangle_{\alpha \beta} \\
& +\left[\left(k_{1}+k_{2}\right)_{\alpha}\left(k_{1}+k_{2}\right)_{\beta}\right] \triangle_{\alpha \beta},
\end{aligned}
$$

where the last term is, in principle, ambiguous. The finite contributions and that proportional to the basic divergent integral $I_{l o g}$ are unambiguous since the combination $k_{2}-k_{1}$ is just equal to the external momentum.

With the same ingredients, rigorously, we can calculate the pseudoscalar-pseudoscalar two-point function:

$$
\begin{aligned}
T^{P P}=\int & \frac{d^{4} k}{(2 \pi)^{4}} \operatorname{Tr}\left\{\gamma_{5} \frac{1}{\left(k+\not k_{1}\right)-m} \gamma_{5} \frac{1}{\left(k+\not k_{2}\right)-m}\right\} \\
=4 & \left\{-I_{\text {quad }}\left(m^{2}\right)+\frac{\left(k_{1}-k_{2}\right)^{2}}{2}\left[I_{\text {log }}\left(m^{2}\right)\right]\right. \\
& \left.-\frac{\left(k_{1}-k_{2}\right)^{2}}{2}\left(\frac{i}{(4 \pi)^{2}}\right) Z_{0}\left(m^{2}, m^{2},\left(k_{1}-k_{2}\right)^{2} ; m^{2}\right)\right\} \\
& -\left[\left(k_{1}-k_{2}\right)_{\alpha}\left(k_{1}-k_{2}\right)_{\beta}\right] \triangle_{\alpha \beta} \\
& -\left[\left(k_{1}+k_{2}\right)_{\alpha}\left(k_{1}+k_{2}\right)_{\beta}\right] \triangle_{\alpha \beta},
\end{aligned}
$$


which also presents a potentially ambiguous term. Now, for the evaluation of the $T_{\mu}^{A P}$ two-point function only the result in eq.(69) is needed. After the traces calculations:

$$
\begin{aligned}
T_{\mu}^{A P} & =\int \frac{d^{4} k}{(2 \pi)^{4}} \operatorname{Tr}\left\{i \gamma_{\mu} \gamma_{5} \frac{1}{\left(k+\not k_{1}\right)-m} \gamma_{5} \frac{1}{\left(\not k+\not k_{2}\right)-m}\right\} \\
& =-4 m i\left(k_{1}-k_{2}\right)_{\mu}\left\{\left[I_{l o g}\left(m^{2}\right)\right]-\left(\frac{i}{(4 \pi)^{2}}\right) Z_{0}\left(m^{2}, m^{2},\left(k_{1}-k_{2}\right)^{2} ; m^{2}\right)\right\},
\end{aligned}
$$

which is unambiguous. Following the calculations we consider the vector-scalar two-point function, defined as

$$
T_{\mu}^{V S}=\int \frac{d^{4} k}{(2 \pi)^{4}} \operatorname{Tr}\left\{\gamma_{\mu} \frac{1}{\left(\not k+k_{1}\right)-m} \hat{1} \frac{1}{\left(k+\not k_{2}\right)-m}\right\}
$$

which, solving the trace operation, can be written as

$$
\begin{aligned}
T_{\mu}^{V S}= & 4 m\left\{\int \frac{d^{4} k}{(2 \pi)^{4}} \frac{2 k_{\mu}}{\left[\left(k+k_{1}\right)^{2}-m^{2}\right]\left[\left(k+k_{2}\right)^{2}-m^{2}\right]}\right. \\
& \left.+\left(k_{1}+k_{2}\right)_{\mu} \int \frac{d^{4} k}{(2 \pi)^{4}} \frac{1}{\left[\left(k+k_{1}\right)^{2}-m^{2}\right]\left[\left(k+k_{2}\right)^{2}-m^{2}\right]}\right\},
\end{aligned}
$$

where a new type of divergent integral, linearly divergent, have appeared.

Following our recipe, we first use the identity eq.(49) with $N=1$ for both terms in the integral to obtain

$$
\begin{aligned}
\int_{\Lambda} \frac{d^{4} k}{(2 \pi)^{4}} \frac{k_{\mu}}{\left[\left(k+k_{1}\right)^{2}-m^{2}\right]\left[\left(k+k_{2}\right)^{2}-m^{2}\right]}= & -\frac{\left(k_{1}+k_{2}\right)_{\alpha}}{2} \int_{\Lambda} \frac{d^{4} k}{(2 \pi)^{4}} \frac{4 k_{\alpha} k_{\mu}}{\left(k^{2}-m^{2}\right)^{3}} \\
& +\int \frac{d^{4} k}{(2 \pi)^{4}} \frac{\left(k_{1}^{2}+2 k_{1} \cdot k\right)^{2} k_{\mu}}{\left[\left(k^{2}-m^{2}\right)^{3}\right]\left[\left(k+k_{1}\right)^{2}-m^{2}\right]} \\
& +\int \frac{d^{4} k}{(2 \pi)^{4}} \frac{\left(k_{2}^{2}+2 k_{2} \cdot k\right)^{2} k_{\mu}}{\left[\left(k^{2}-m^{2}\right)^{3}\right]\left[\left(k+k_{2}\right)^{2}-m^{2}\right]} \\
& +\int \frac{d^{4} k}{(2 \pi)^{4}} \frac{\left(k_{1}^{2}+2 k_{1} \cdot k\right)\left(k_{2}^{2}+2 k_{2} \cdot k\right) k_{\mu}}{\left[\left(k^{2}-m^{2}\right)^{2}\right]\left[\left(k+k_{1}\right)^{2}-m^{2}\right]\left[\left(k+k_{2}\right)^{2}-m^{2}\right]} .
\end{aligned}
$$

Only an odd integral have been removed on the right hand side of the above equation, in addition to the subscript $\Lambda$ on the last three (finite) integrals, which, after the integration, produces the results;

$$
\begin{gathered}
\text { - } \int \frac{d^{4} k}{(2 \pi)^{4}} \frac{\left(k_{1}^{2}+2 k_{1} \cdot k\right)^{2} k_{\mu}}{\left[\left(k^{2}-m^{2}\right)^{3}\right]\left[\left(k+k_{1}\right)^{2}-m^{2}\right]}=\left(\frac{i}{(4 \pi)^{2}}\right)\left[k_{1 \mu}\right]\left[Z_{1}\left(m^{2}, m^{2}, k_{1}^{2} ; m^{2}\right)\right] \\
\qquad \int \frac{d^{4} k}{(2 \pi)^{4}} \frac{\left(k_{1}^{2}+2 k_{1} \cdot k\right)\left(k_{2}^{2}+2 k_{2} \cdot k\right) k_{\mu}}{\left[\left(k^{2}-m^{2}\right)^{2}\right]\left[\left(k+k_{1}\right)^{2}-m^{2}\right]\left[\left(k+k_{2}\right)^{2}-m^{2}\right]}= \\
=\left(\frac{i}{(4 \pi)^{2}}\right)\left[-k_{1 \mu} Z_{1}\left(m^{2}, m^{2}, k_{1}^{2} ; m^{2}\right)-k_{2 \mu} Z_{1}\left(m^{2}, m^{2}, k_{2}^{2} ; m^{2}\right)\right. \\
\left.+\left(k_{1}+k_{2}\right)_{\mu} Z_{1}\left(m^{2}, m^{2},\left(k_{1}-k_{2}\right)^{2} ; m^{2}\right)\right]
\end{gathered}
$$

If we consider also a property of the $Z_{k}$ functions

$$
Z_{1}\left(m^{2}, m^{2}, q^{2} ; m^{2}\right)=\frac{Z_{0}\left(m^{2}, m^{2}, q^{2} ; m^{2}\right)}{2}
$$

we can write the result for the linearly divergent integral as:

$$
\begin{gathered}
\int_{\Lambda} \frac{d^{4} k}{(2 \pi)^{4}} \frac{k_{\mu}}{\left[\left(k+k_{1}\right)^{2}-m^{2}\right]\left[\left(k+k_{2}\right)^{2}-m^{2}\right]}=-\frac{\left(k_{1}+k_{2}\right)_{\alpha}}{2} \int_{\Lambda} \frac{d^{4} k}{(2 \pi)^{4}} \frac{4 k_{\mu} k_{\alpha}}{\left(k^{2}-m^{2}\right)^{3}} \\
+\left(\frac{i}{(4 \pi)^{2}}\right) \frac{\left(k_{1}+k_{2}\right)_{\mu}}{2}\left[Z_{0}\left(m^{2}, m^{2},\left(k_{1}-k_{2}\right)^{2} ; m^{2}\right)\right] .
\end{gathered}
$$


Then, after the substitution of the eqs.(69) and (79) results, we get for the $T_{\mu}^{V S}$ amplitude

$$
T_{\mu}^{V S}=(-) 4 m\left(k_{1}+k_{2}\right)_{\beta}\left[\triangle_{\beta \mu}\right] .
$$

Let us now consider those two-point functions with two Lorentz indexes. First the one which play a crucial role in our investigation, as become clear in what follows, namely the axial-vector-vector, defined by

$$
T_{\mu \nu}^{A V}=\int \frac{d^{4} k}{(2 \pi)^{4}} \operatorname{Tr}\left\{i \gamma_{\mu} \gamma_{5} \frac{1}{\left(k+\not k_{1}\right)-m} \gamma_{\nu} \frac{1}{\left(k+\not k_{2}\right)-m}\right\}
$$

Solving the traces involved;

$$
\begin{aligned}
T_{\mu \nu}^{A V}= & -4 \varepsilon_{\mu \nu \alpha \beta}\left\{k_{2 \beta} \int \frac{d^{4} k}{(2 \pi)^{4}} \frac{k_{\alpha}}{\left[\left(k+k_{1}\right)^{2}-m^{2}\right]\left[\left(k+k_{2}\right)^{2}-m^{2}\right]}\right. \\
& +k_{1 \alpha} \int \frac{d^{4} k}{(2 \pi)^{4}} \frac{k_{\beta}}{\left[\left(k+k_{1}\right)^{2}-m^{2}\right]\left[\left(k+k_{2}\right)^{2}-m^{2}\right]} \\
& \left.+k_{1 \alpha} k_{2 \beta} \int \frac{d^{4} k}{(2 \pi)^{4}} \frac{1}{\left[\left(k+k_{1}\right)^{2}-m^{2}\right]\left[\left(k+k_{2}\right)^{2}-m^{2}\right]}\right\} .
\end{aligned}
$$

To complete the calculations only the results eq.(69) and Eq.(79), the same ones used in the $T_{\mu}^{V S}$ calculations, are needed. We obtain then

$$
T_{\mu \nu}^{A V}=2 \varepsilon_{\mu \nu \alpha \beta}\left[\left(k_{2}-k_{1}\right)_{\beta}\left(k_{1}+k_{2}\right)_{\xi} \triangle_{\xi \alpha}\right] .
$$

Note that this amplitude constitutes the first pseudo one considered at this point. For while we call attention for the fact that no special procedures have been adopted.

Remain to evaluate only two amplitudes, the more complex ones. First we take the $T_{\mu \nu}^{V V}$ amplitude defined by

$$
T_{\mu \nu}^{V V}=\int \frac{d^{4} k}{(2 \pi)^{4}} \operatorname{Tr}\left\{\gamma_{\mu} \frac{1}{\left(\not k+\not k_{1}\right)-m} \gamma_{\nu} \frac{1}{\left(\not k+\not k_{2}\right)-m}\right\} .
$$

The traces evaluations lead us to write,

$$
T_{\mu \nu}^{V V}=T_{\mu \nu}+g_{\mu \nu}\left[T^{P P}\right],
$$

where the term proportional to $g_{\mu \nu}$, coming from the trace, is an identical mathematical structure to the $T^{P P}$ twopoint function, in such a way the same results will be adopted, and the tensor $T_{\mu \nu}$ is defined by

$$
T_{\mu \nu}=4 \int \frac{d^{4} k}{(2 \pi)^{4}} \frac{\left[\left(k+k_{1}\right)_{\mu}\left(k+k_{2}\right)_{\nu}+\left(k+k_{1}\right)_{\nu}\left(k+k_{2}\right)_{\mu}\right]}{\left[\left(k+k_{1}\right)^{2}-m^{2}\right]\left[\left(k+k_{2}\right)^{2}-m^{2}\right]} .
$$

To evaluate $T_{\mu \nu}$ we first need to consider the quadratically divergent integral present in the above expression. Following strictly the same procedure adopted on the evaluation of the logarithmically and linearly divergent cases, choosing adequate values for $N$ in the identity eq.(49), after a long and tedious calculations, we will arrive to the result

$$
\begin{aligned}
\int_{\Lambda} \frac{d^{4} k}{(2 \pi)^{4}} \frac{k_{\mu} k_{\nu}}{\left[\left(k+k_{1}\right)^{2}-m^{2}\right]\left[\left(k+k_{2}\right)^{2}-m^{2}\right]}= \\
\quad\left\{\int_{\Lambda} \frac{d^{4} k}{(2 \pi)^{4}} \frac{k_{\mu} k_{\nu}}{\left[\left(k^{2}-m^{2}\right)^{2}\right.}\right\} \\
+\left\{-\left(k_{1}^{2}+k_{2}^{2}\right) \int_{\Lambda} \frac{d^{4} k}{(2 \pi)^{4}} \frac{k_{\mu} k_{\nu}}{\left[\left(k^{2}-m^{2}\right)^{3}\right.}+\left(k_{1 \xi} k_{1 \beta}+k_{2 \xi} k_{2 \beta}+k_{1 \beta} k_{2 \xi}\right) \int_{\Lambda} \frac{d^{4} k}{(2 \pi)^{4}} \frac{4 k_{\xi} k_{\beta} k_{\mu} k_{\nu}}{\left[\left(k^{2}-m^{2}\right)^{4}\right.}\right\} \\
+\left(\frac{i}{(4 \pi)^{2}}\right)\left\{\left[\left(k_{1}-k_{2}\right)_{\mu}\left(k_{1}-k_{2}\right)_{\nu}-\left(k_{1}-k_{2}\right)^{2} g_{\mu \nu}\right] \times\right. \\
\times\left[-Z_{2}\left(m^{2}, m^{2},\left(k_{1}-k_{2}\right)^{2} ; m^{2}\right)+\frac{Z_{0}\left(m^{2}, m^{2},\left(k_{1}-k_{2}\right)^{2} ; m^{2}\right)}{4}\right] \\
\left.\quad-\frac{\left(k_{1}+k_{2}\right)_{\mu}\left(k_{1}+k_{2}\right)_{\nu}}{4} Z_{0}\left(m^{2}, m^{2},\left(k_{1}-k_{2}\right)^{2} ; m^{2}\right)\right\} .
\end{aligned}
$$


With this result in addition to eq.(69), eq.(79), eq.(85), eq.(71) and the property of the $Z_{k}$ functions

$$
Z_{2}\left(m^{2}, m^{2}, q^{2} ; m^{2}\right)=-\frac{1}{18}-\frac{m^{2}}{3 q^{2}} Z_{0}\left(m^{2}, m^{2}, q^{2} ; m^{2}\right)+\frac{Z_{0}\left(m^{2}, m^{2}, q^{2} ; m^{2}\right)}{3},
$$

a very useful expression for $T_{\mu \nu}$ can be obtained

$$
\begin{aligned}
T_{\mu \nu}= & \frac{4}{3}\left[\left(k_{1}-k_{2}\right)^{2} g_{\mu \nu}-\left(k_{1}-k_{2}\right)_{\mu}\left(k_{1}-k_{2}\right)_{\nu}\right] \times \\
& \times\left\{I_{l o g}\left(m^{2}\right)-\left(\frac{i}{(4 \pi)^{2}}\right)\left[\frac{1}{3}+\left(\frac{2 m^{2}+\left(k_{1}-k_{2}\right)^{2}}{\left(k_{1}-k_{2}\right)^{2}}\right) Z_{0}\left(m^{2}, m^{2},\left(k_{1}-k_{2}\right)^{2} ; m^{2}\right)\right]\right\} \\
& -\left[T^{P P}\right] g_{\mu \nu}+A_{\mu \nu},
\end{aligned}
$$

where

$$
\begin{aligned}
A_{\mu \nu}= & 4\left[\nabla_{\mu \nu}\right]+\left(k_{1}-k_{2}\right)_{\alpha}\left(k_{1}-k_{2}\right)_{\beta}\left[\frac{1}{3} \square_{\alpha \beta \mu \nu}+\frac{1}{3} \triangle_{\mu \beta} g_{\alpha \nu}+g_{\alpha \mu} \triangle_{\beta \nu}-g_{\mu \nu} \triangle_{\alpha \beta}-\frac{2}{3} g_{\alpha \beta} \triangle_{\mu \nu}\right] \\
& +\left[\left(k_{1}-k_{2}\right)_{\alpha}\left(k_{1}+k_{2}\right)_{\beta}-\left(k_{1}+k_{2}\right)_{\alpha}\left(k_{1}-k_{2}\right)_{\beta}\right]\left[\frac{1}{3} \square_{\alpha \beta \mu \nu}+\frac{1}{3} \triangle_{\mu \beta} g_{\nu \alpha}+\frac{1}{3} \triangle_{\beta \nu} g_{\alpha \mu}\right] \\
& +\left(k_{1}+k_{2}\right)_{\alpha}\left(k_{1}+k_{2}\right)_{\beta}\left[\square_{\alpha \beta \mu \nu}-\triangle_{\nu \alpha} g_{\mu \beta}-\triangle_{\beta \nu} g_{\alpha \mu}-3 \triangle_{\alpha \beta} g_{\mu \nu}\right] .
\end{aligned}
$$

Then we are at the position to write the expression for the $T_{\mu \nu}^{V V}$ amplitude

$$
\begin{aligned}
T_{\mu \nu}^{V V}= & \frac{4}{3}\left[\left(k_{1}-k_{2}\right)^{2} g_{\mu \nu}-\left(k_{1}-k_{2}\right)_{\mu}\left(k_{1}-k_{2}\right)_{\nu}\right] \times \\
& \times\left\{\left[I_{l o g}\left(m^{2}\right)\right]-\left(\frac{i}{(4 \pi)^{2}}\right)\left[\frac{1}{3}+\frac{\left(2 m^{2}+\left(k_{1}-k_{2}\right)^{2}\right)}{\left(k_{1}-k_{2}\right)^{2}} Z_{0}\left(m^{2}, m^{2},\left(k_{1}-k_{2}\right)^{2} ; m^{2}\right)\right]\right\}+A_{\mu \nu} .
\end{aligned}
$$

Finally we consider the $A A$ two-point function,

$$
T_{\mu \nu}^{A A}=\int \frac{d^{4} k}{(2 \pi)^{4}} \operatorname{Tr}\left\{i \gamma_{\mu} \gamma_{5} \frac{1}{[k+\not k+m]} i \gamma_{\nu} \gamma_{5} \frac{1}{\left[\not k+\not k_{2}-m\right]}\right\}
$$

which exhibits the structure

$$
T_{\mu \nu}^{A A}=g_{\mu \nu}\left[T^{S S}\right]-T_{\mu \nu}
$$

After the traces evaluation is performed. No additional calculations are needed if we identify the relation between two-point functions

$$
\left[T^{S S}+T^{P P}\right]=\frac{2 m i\left(k_{1}-k_{2}\right)_{\mu}}{\left(k_{1}-k_{2}\right)^{2}} T_{\mu}^{A P}
$$

collecting the results eq.(89), eq.(94), and substituting in eq.(93), the expression for $T_{\mu \nu}^{A A}$ amplitude so obtained is:

$$
\begin{aligned}
T_{\mu \nu}^{A A}= & -\frac{4}{3}\left[\left(k_{1}-k_{2}\right)^{2} g_{\mu \nu}-\left(k_{1}-k_{2}\right)_{\mu}\left(k_{1}-k_{2}\right)_{\nu}\right] \times \\
& \times\left\{\left[I_{l o g}\left(m^{2}\right)\right]-\left(\frac{i}{(4 \pi)^{2}}\right)\left[\frac{1}{3}+\frac{\left(2 m^{2}+\left(k_{1}-k_{2}\right)^{2}\right)}{\left(k_{1}-k_{2}\right)^{2}} Z_{0}\left(m^{2}, m^{2},\left(k_{1}-k_{2}\right)^{2} ; m^{2}\right)\right]\right\} \\
& +g_{\mu \nu} 8 m^{2}\left\{\left[I_{l o g}\left(m^{2}\right)\right]-\left(\frac{i}{(4 \pi)^{2}}\right) Z_{0}\left(m^{2}, m^{2},\left(k_{1}-k_{2}\right)^{2} ; m^{2}\right)\right\}-A_{\mu \nu .} .
\end{aligned}
$$

This calculation completes the evaluation of all non-vanishing two-point functions. It is a simple matter to show that the remaining ones $T_{\mu}^{V P}, T^{P S}$ and $T_{\mu}^{A S}$ vanishes identically due to the presence of the $\gamma_{5}$ matrix. 


\section{Three Point Functions}

We now come to the three-point functions. In order not to overload the text we limit ourselves in writing, in the explicit way, only the terms indicating possibility of ambiguities. This is sufficient for the analysis we want to do, closely related to that performed in ref. [1]. To illustrate what we mean, consider a relatively simple case of three-point functions:

$$
T_{\lambda}^{V S S}=\int \frac{d^{4} k}{(2 \pi)^{4}} \operatorname{Tr}\left\{\gamma_{\lambda} \frac{1}{\left[k+\not k_{3}-m\right]} \hat{1} \frac{1}{\left[\not k+\not k_{2}-m\right]} \hat{1} \frac{1}{\left[\not k+\not k_{1}-m\right]}\right\}
$$

After taking the trace and performing some standard algebraic reorganization of terms we obtain the expression

$$
\begin{aligned}
T_{\lambda}^{V S S}= & 2\left\{\int \frac{d^{4} k}{(2 \pi)^{4}} \frac{2 k_{\lambda}}{\left[\left(k+k_{2}\right)^{2}-m^{2}\right]\left[\left(k+k_{3}\right)^{2}-m^{2}\right]}\right. \\
+ & \left(k_{2}+k_{3}\right)_{\lambda} \int \frac{d^{4} k}{(2 \pi)^{4}} \frac{1}{\left[\left(k+k_{2}\right)^{2}-m^{2}\right]\left[\left(k+k_{3}\right)^{2}-m^{2}\right]} \\
+ & \left(k_{3}-k_{1}\right)_{\lambda} \int \frac{d^{4} k}{(2 \pi)^{4}} \frac{1}{\left[\left(k+k_{1}\right)^{2}-m^{2}\right]\left[\left(k+k_{3}\right)^{2}-m^{2}\right]} \\
+ & \left(k_{2}-k_{1}\right)_{\lambda} \int \frac{d^{4} k}{(2 \pi)^{4}} \frac{1}{\left[\left(k+k_{1}\right)^{2}-m^{2}\right]\left[\left(k+k_{2}\right)^{2}-m^{2}\right]} \\
+ & {\left[8 m^{2}-\left(k_{1}-k_{2}\right)^{2}-\left(k_{1}-k_{3}\right)^{2}+\left(k_{2}-k_{3}\right)^{2}\right] \times } \\
& \times \int \frac{d^{4} k}{(2 \pi)^{4}} \frac{k_{\lambda}}{\left[k^{2}-m^{2}\right]\left[\left(k+k_{2}-k_{1}\right)^{2}-m^{2}\right]\left[\left(k+k_{3}-k_{1}\right)^{2}-m^{2}\right]} \\
+ & {\left[\left[4 m^{2}-\left(k_{1}-k_{2}\right)^{2}\right]\left(k_{3}-k_{1}\right)_{\lambda}+\left[4 m^{2}-\left(k_{1}-k_{3}\right)^{2}\right]\left(k_{2}-k_{1}\right)_{\lambda}\right] \times } \\
& \left.\times \int \frac{d^{4} k}{(2 \pi)^{4}} \frac{1}{\left[k^{2}-m^{2}\right]\left[\left(k+k_{2}-k_{1}\right)^{2}-m^{2}\right]\left[\left(k+k_{3}-k_{1}\right)^{2}-m^{2}\right]}\right\} .
\end{aligned}
$$

In the above expression we note that only the first two terms are potentially ambiguous. The remaining ones are either logarithmically divergent or finite, all with unambiguous coefficients. Of course it is possible to solve all integrals and obtain an analytical expression for $T_{\lambda}^{V S S}[19]$. But, for our immediate purposes, it is enough to write it in the form

$$
T_{\lambda}^{V S S}=-2\left(k_{2}+k_{3}\right)_{\xi}\left[\triangle_{\lambda \xi}\right]+N A T
$$

where NAT stands for non-ambiguous terms. Having this in mind we simply list the other three-point functions in the same manner;

$$
T_{\lambda}^{V P P}=2\left(k_{2}+k_{3}\right)_{\xi}\left[\triangle_{\lambda \xi}\right]+N A T,
$$

with only one Lorentz index we have also

$$
T_{\mu}^{S A P}=2 i\left(k_{1}+k_{3}\right)_{\xi}\left[\triangle_{\mu \xi}\right]+N A T .
$$

On the other hand, with three Lorentz indexes;

$$
\begin{gathered}
T_{\lambda \mu \nu}^{A A A}=-2 \varepsilon_{\lambda \mu \nu \xi}\left(k_{1}+k_{2}\right)_{\sigma}\left(\triangle_{\xi \sigma}\right)+N A T, \\
T_{\lambda \mu \nu}^{A V V}=2 \varepsilon_{\lambda \mu \nu \xi}\left(k_{1}+k_{2}\right)_{\sigma}\left(\triangle_{\xi \sigma}\right)+N A T
\end{gathered}
$$

and

$$
\begin{aligned}
T_{\lambda \mu \nu}^{V V V}= & \left\{\left(k_{1}+k_{2}\right)_{\xi}\left[-\frac{2}{3} \square_{\xi \mu \nu \lambda}-\frac{2}{3} g_{\xi \nu} \triangle_{\mu \lambda}-\frac{2}{3} g_{\xi \mu} \triangle_{\nu \lambda}-\frac{2}{3} g_{\xi \lambda} \triangle_{\mu \nu}+2 g_{\lambda \mu} \triangle_{\nu \xi}+2 g_{\xi \nu} \triangle_{\lambda \mu}\right]\right. \\
& +\left(k_{1}+k_{3}\right)_{\xi}\left[-\frac{2}{3} \square_{\xi \mu \nu \lambda}-\frac{2}{3} g_{\xi \nu} \triangle_{\mu \lambda}-\frac{2}{3} g_{\xi \mu} \triangle_{\nu \lambda}-\frac{2}{3} g_{\xi \lambda} \triangle_{\mu \nu}+2 g_{\lambda \nu} \triangle_{\mu \xi}+2 g_{\xi \mu} \triangle_{\lambda \nu}\right] \\
& \left.+\left(k_{2}+k_{3}\right)_{\xi}\left[-\frac{2}{3} \square_{\xi \mu \nu \lambda}-\frac{2}{3} g_{\xi \nu} \triangle_{\mu \lambda}-\frac{2}{3} g_{\xi \mu} \triangle_{\nu \lambda}-\frac{2}{3} g_{\xi \lambda} \triangle_{\mu \nu}+2 g_{\mu \nu} \triangle_{\lambda \xi}+2 g_{\xi \lambda} \triangle_{\mu \nu}\right]+N A T\right\} .
\end{aligned}
$$


In order to give a complete account of the three-point functions we give the amplitude $T_{\lambda \mu \nu}^{V A A}$, which possesses the same potentially ambiguous term (AT) as $T_{\lambda \mu \nu}^{V V V}$, i.e.

$$
\left.T_{\lambda \mu \nu}^{V A A}\right|_{A T}=-\left.T_{\lambda \mu \nu}^{V V V}\right|_{A T} .
$$

The remaining three-point functions are all unambiguous, although divergent, therefore are left out of the discussions.

Let us now establish contact between our results and those obtained by Gertsein and Jackiw showing that it is a simple matter to go from our formalism over to theirs.

\section{AMBIGUITIES IN GERTSEIN AND JACKIW APPROACH}

Let us now understand how the results obtained in the previous section include those of ref. [1] as a special case. We show that starting from our expressions we can obtain the results in tables I and II for the ambiguities in the two and three point functions as defined by those authors in section III of ref. [1]. We start with our results for the amplitude $T_{\mu}^{V S}$ given by eq.(80). In the adopted notation the ambiguity has been introduced via the arbitrary momenta $k_{1}$ and $k_{2}$, by calculating the amplitudes with internal momenta in the loop: $k+k_{1}$ and $k+k_{2}$ in the two propagators in question, as defined in eq.(6). In ref. [1] the arbitrariness is represented by the momentum $s$, such that the propagators have momenta $k+s$ and $k+s+p$ which establish the equivalent relation

$$
\left\{\begin{array}{c}
k_{1}=s \\
k_{2}=s+p
\end{array}\right.
$$

In this way, the ambiguous part of the $T_{\mu}^{V S}$ amplitude can be cast into the form

$$
T_{\mu}^{V S}=-4 m(2 s)_{\beta}\left\{\int_{\Lambda} \frac{d^{4} k}{(2 \pi)^{4}} \frac{4 k_{\mu} k_{\beta}}{\left(k^{2}-m^{2}\right)^{3}}-\int_{\Lambda} \frac{d^{4} k}{(2 \pi)^{4}} \frac{g_{\mu \beta}}{\left(k^{2}-m^{2}\right)^{2}}\right\},
$$

where we have used the definition in eq.(51) for the object $\triangle_{\beta \mu}$. Taking now the limit in a symmetric way in the first integral, i.e., using the relation

$$
\lim _{k \rightarrow \infty} k_{\mu} k_{\beta}=\frac{1}{4} k^{2} g_{\mu \beta}
$$

and taking $k^{2} \rightarrow k^{2}-m^{2}+m^{2}$ in this integral we get

$$
T_{\mu}^{V S}=-4 m(2 s)_{\beta}\left\{\int \frac{d^{4} k}{(2 \pi)^{4}} \frac{m^{2} g_{\mu \beta}}{\left(k^{2}-m^{2}\right)^{3}}\right\} .
$$

Using the result

$$
\int \frac{d^{4} k}{(2 \pi)^{4}} \frac{1}{\left(k^{2}-m^{2}\right)^{3}}=\frac{i}{2(4 \pi)^{2}\left(-m^{2}\right)}
$$

we finally get

$$
T_{\mu}^{V S}=\frac{4 i \pi^{2}}{(2 \pi)^{4}} m s_{\mu},
$$

as in ref. [1]. In an analogous way we get for the ambiguous contribution of $T^{P P}$ and $T^{S S}$

$$
\left.T^{P P}\right|_{A T}=\left.T^{S S}\right|_{A T}=\frac{-i \pi^{2}}{(2 \pi)^{4}} s \cdot(s+p),
$$

and for $T_{\mu \nu}^{A V}$

$$
T_{\mu \nu}^{A V}=\frac{-2 i \pi^{2} \varepsilon_{\mu \alpha \nu \beta} s_{\alpha} p_{\beta}}{(2 \pi)^{4}} .
$$


The functions $T_{\mu \nu}^{V V}$ and $T_{\mu \nu}^{A A}$ have the ambiguous part given by $A_{\mu \nu}$ in eq.(90). An evaluation for $\square_{\alpha \beta \mu \nu}$, defined in eq.(50), is in order. It is necessary to use the relation

$$
\lim _{k \rightarrow \infty} \frac{k_{\alpha} k_{\beta} k_{\mu} k_{\nu}}{k^{4}}=\frac{1}{24}\left(g_{\alpha \beta} g_{\mu \nu}+g_{\alpha \mu} g_{\beta \nu}+g_{\alpha \nu} g_{\beta \mu}\right)
$$

and also the following results

$$
\int \frac{d^{4} k}{(2 \pi)^{4}} \frac{k_{\mu} k_{\nu}}{\left(k^{2}-m^{2}\right)^{4}}=\frac{i}{(4 \pi)^{2}} \frac{1 / 2 g_{\mu \nu}}{3 !\left(-m^{2}\right)}
$$

and

$$
\int \frac{d^{4} k}{(2 \pi)^{4}} \frac{k^{2}}{\left(k^{2}-m^{2}\right)^{4}}=\frac{i}{(4 \pi)^{2}} \frac{1}{3\left(-m^{2}\right)}
$$

in order to obtain (after some algebra)

$$
\left.T_{\mu \nu}^{V V}\right|_{A T}=\left.T_{\mu \nu}^{A A}\right|_{A T}=\frac{2 i \pi^{2}}{3(2 \pi)^{4}}\left\{s_{\mu}(s+p)_{\nu}+g_{\mu \nu} s \cdot(s+p)+s_{\nu}(s+p)_{\mu}\right\} .
$$

In the case of the three-point functions, in order to obtain the results of ref. [1] it is necessary to take the $\mathrm{SU}(3)$ indices into account in the calculation of the amplitudes, since they play an important role in the construction of currents. The Gell-Mann matrices introduce symmetry factors such as relative global signs between the direct and crossed channel, which are not present in our non-abelian model. However this does not introduce any inconsistency in the two formulations, as we will see.

The arbitrariness in the label for the internal momenta of the loop is introduced by taking

$$
s=a p+b p^{\prime},
$$

where $a$ and $b$ are real numbers. The ambiguity is then found by means of calculating the difference between two representations for the three-point functions: with and without the arbitrary label $s$ defined above. In this sense we see that it can be interpreted as a surface term, which would disappear if shifts in the integration variable were allowed.

Following this prescription, the ambiguous term of $T_{\mu}^{S A P}$ previously found in the form

$$
\left.T_{\mu}^{S A P}\right|_{A T}=2 i\left(k_{1}+k_{3}\right)_{\xi} \triangle_{\mu \xi}
$$

for the direct channel (without $\mathrm{SU}(3)$ factors) may be written as

$$
\left.T_{\mu}^{S A P}\right|_{A T}=\frac{2 \pi^{2}}{(2 \pi)^{4}}\left(a p+b p^{\prime}\right)_{\mu}
$$

which, after the inclusion of the crossed channel and the pertinent factors yield

$$
\left.T_{\mu}^{S \rightarrow A P}\right|_{A T}=\frac{2 \pi^{2}}{(2 \pi)^{4}}(a-b)\left(p-p^{\prime}\right)_{\mu} .
$$

In a completely analogous way we get

$$
\begin{gathered}
\left.T_{\mu}^{V \rightarrow S S}\right|_{A T}=\left.T_{\mu}^{V \rightarrow P P}\right|_{A T}=\frac{-i 2 \pi^{2}}{(2 \pi)^{4}}(a-b)\left(p-p^{\prime}\right)_{\mu}, \\
\left.T_{\mu \alpha \beta}^{A \rightarrow A A}\right|_{A T}=\left.T_{\mu \alpha \beta}^{A \rightarrow V V}\right|_{A T}=\frac{-2 \pi^{2}}{(2 \pi)^{4}}(b-a) \varepsilon_{\mu \alpha \beta \lambda}\left(p-p^{\prime}\right)_{\lambda},
\end{gathered}
$$

and finally

$$
\left.T_{\mu \alpha \beta}^{V \rightarrow V V}\right|_{A T}=\left.T_{\mu \alpha \beta}^{V \rightarrow A A}\right|_{A T}=\frac{-2 i \pi^{2}}{3(2 \pi)^{4}}(b-a)\left\{g_{\mu \alpha}\left(p-p^{\prime}\right)_{\beta}+g_{\mu \beta}\left(p-p^{\prime}\right)_{\alpha}+g_{\alpha \beta}(p-q)_{\mu}\right\} .
$$

The above demonstrations show us, as enounced, that our calculational technique produces results mappeable in others specific procedures. Let us now verify the Ward Identities involved. 


\section{WARD IDENTITIES}

We next proceed to the verification of the free fermion model symmetry relations presented by eqs.(12)-(47). Such relations were obtained formally by contracting the Lorentz index of a vertex with the respective external momentum. There are in principle two ways to verify Ward Identities involving n-point functions. The first is to contract the integral representation, before evaluating traces, generating in this way a function with the same number of points as the contracted one more two others with a number of points lowered by one unity. Only the former ones are in fact evaluated explicitly [19]. This is the strategy used in ref. [1] to analyze Ward Identities. The second way involves the explicitly evaluation of the n-point function in question and afterwards contracting the results with the appropriate external momentum, identifying then all others functions present in the investigated Ward Identities. Obviously the effort associated is much higher in the second option once all functions involved needs to be evaluated explicitly. We will analyze in this work both possibilities for two-point functions and only the first one for the three-point functions.

\section{A. One-Point Functions}

The first Ward Identity to which we refer in section II involves $T_{\mu}^{V}$. A vanishing value for this amplitude is predicted in QED by Furry's theorem, for example. From the result produced by our treatment, eq.(59), we see that in order to obtain a definite zero value for the $T_{\mu}^{V}$ amplitude in such a way that the result is independent of the arbitrary choices for the internal momentum the following conditions need to be satisfied:

$$
\left\{\begin{array}{l}
\square_{\alpha \beta \mu \nu}=0 \\
\nabla_{\mu \nu}=0 \\
\triangle_{\mu \nu}=0 .
\end{array}\right.
$$

Which we will denominate consistency conditions [24] from now on. Since all terms of $T_{\mu}^{V}$ in eq.(59) are ambiguous, requiring relations eqs.(122) the amplitude turns unambiguous and symmetry preserving. As will be shown in what follows they are necessary and sufficient conditions to "save" all other amplitudes from ambiguities and symmetry violations.

\section{B. Two-Point Functions}

We now study the Ward Identities for the two-point functions. Let us, initially, consider the contraction of $T_{\mu}^{V S}$ with the external momentum $\left(k_{1}-k_{2}\right)_{\mu}$

$$
\left(k_{1}-k_{2}\right)_{\mu} T_{\mu}^{V S}=\int \frac{d^{4} k}{(2 \pi)^{4}} \operatorname{Tr}\left\{\hat{1} \frac{1}{\left[\not k+\not k_{2}-m\right]}\left(k_{1}-\not k_{2}\right) \frac{1}{\left[\not k+\not k_{1}-m\right]}\right\} .
$$

Before calculating the trace we use the following identity

$$
\left(\not k_{1}-\not k_{2}\right)=\left[\not k+\not k_{1}-m\right]-\left[\not k+\not k_{2}-m\right]
$$

so that

$$
\left(k_{1}-k_{2}\right)_{\mu} T_{\mu}^{V S}=\int \frac{d^{4} k}{(2 \pi)^{4}} \operatorname{Tr}\left\{\hat{1} \frac{1}{\left[k+\not k_{2}-m\right]}\right\}-\int \frac{d^{4} k}{(2 \pi)^{4}} \operatorname{Tr}\left\{\hat{1} \frac{1}{\left[\not k+\not k_{1}-m\right]}\right\} .
$$

Comparing now with eq.(60) we identify two scalar one-point functions. It is clear that the vector current will only be conserved if there is no dependence on $k_{1}$ and $k_{2}$ in this one-point functions. However, using the result in eq.(62) for $T^{S}$ we get

$$
\left(k_{1}-k_{2}\right)_{\mu} T_{\mu}^{V S}=-2 m\left[\left(k_{1}+k_{2}\right)_{\alpha}\left(k_{1}-k_{2}\right)_{\beta}+\left(k_{1}-k_{2}\right)_{\alpha}\left(k_{1}+k_{2}\right)_{\beta}\right] \triangle_{\alpha \beta} .
$$

We can, on the other hand, also check the validity of the identity by the contraction of $\left(k_{1}-k_{2}\right)_{\mu}$ with the expression obtained for $T_{\mu}^{V S}$, eq.(80):

$$
\left(k_{1}-k_{2}\right)_{\mu} T_{\mu}^{V S}=-4 m\left(k_{1}-k_{2}\right)_{\mu}\left(k_{1}+k_{2}\right)_{\beta}\left[\triangle_{\mu \beta}\right] .
$$


In any case the identity can be satisfied provided $\triangle_{\mu \beta}=0$, which renders $T^{S}$ unambiguous and $T_{\mu}^{V S}$ zero, as one can check from eq.(62) and eq.(80). Let us now study the case of axial-pseudoscalar amplitude $T_{\mu}^{A P}$, defined in eq.(72). Firstly we do the following

$$
\left(k_{1}-k_{2}\right)_{\mu} T_{\mu}^{A P}=\int \frac{d^{4} k}{(2 \pi)^{4}} \operatorname{Tr}\left\{\gamma_{5} \frac{1}{\left[k+\not k_{2}-m\right]} i\left(k_{1}-\not k_{2}\right) \gamma_{5} \frac{1}{\left[k+\not k_{1}-m\right]}\right\},
$$

and introduce the identity

$$
\left(k_{2}-\not k_{1}\right) \gamma_{5}=\left[\not k+\not k_{2}-m\right] \gamma_{5}+\gamma_{5}\left[\not k+\not k_{1}-m\right]+2 m \gamma_{5}
$$

in the interior of the trace, which leads us to

$$
\begin{aligned}
\left(k_{1}-k_{2}\right)_{\mu} T_{\mu}^{A P}= & -2 m i T^{P P}-i \int \frac{d^{4} k}{(2 \pi)^{4}} \operatorname{Tr}\left\{\hat{1} \frac{1}{\left[k+\not k_{1}-m\right]}\right\} \\
& -i \int \frac{d^{4} k}{(2 \pi)^{4}} \operatorname{Tr}\left\{\hat{1} \frac{1}{[k+\not k 2-m]}\right\} .
\end{aligned}
$$

Substituting the results previously obtained for the one-point function we obtain

$$
\left(k_{1}-k_{2}\right)_{\mu} T_{\mu}^{A P}=-2 m i T^{P P}-2 m i\left\{4 I_{q u a d}\left(m^{2}\right)+2\left[k_{1 \alpha} k_{1 \beta}+k_{2 \alpha} k_{2 \beta}\right] \triangle_{\alpha \beta}\right\} .
$$

Had we, on the other hand, taken eq.(72) and contracting it with $\left(k_{1}-k_{2}\right)_{\mu}$, we would have gotten

$$
\left(k_{1}-k_{2}\right)_{\mu} T_{\mu}^{A P}=-4 m i\left(k_{1}-k_{2}\right)^{2}\left\{I_{l o g}\left(m^{2}\right)-\left(\frac{i}{(4 \pi)^{2}}\right) Z_{0}\left(m^{2}, m^{2},\left(k_{1}-k_{2}\right)^{2} ; m^{2}\right)\right\}
$$

from which we could get

$$
\begin{aligned}
\left(k_{1}-k_{2}\right)_{\mu} T_{\mu}^{A P}= & -2 m i\left\{-4 I_{\text {quad }}\left(m^{2}\right)+2\left(k_{1}-k_{2}\right)^{2} I_{\text {log }}\left(m^{2}\right)\right. \\
& -\left(\frac{i}{(4 \pi)^{2}}\right)\left(k_{1}-k_{2}\right)^{2} 2 Z_{0}\left(m^{2}, m^{2},\left(k_{1}-k_{2}\right)^{2} ; m^{2}\right) \\
& \left.-2\left(k_{1 \alpha} k_{1 \beta}+k_{2 \alpha} k_{2 \beta}\right) \triangle_{\alpha \beta}\right\} \\
& -2 m i\left\{4 I_{\text {quad }}\left(m^{2}\right)+2\left(k_{1 \alpha} k_{1 \beta}+k_{2 \alpha} k_{2 \beta}\right) \triangle_{\alpha \beta}\right\}
\end{aligned}
$$

This results agrees with eq.(132) provided we identify, in the first term, in curly brackets, the expression for $T^{P P}$, as we can see from eq.(71).

Let us next take the amplitude $T_{\mu \nu}^{A V}$ which has two Lorentz indices and has two Ward Identities connected to it, one for the vector index and other for the axial one. For the vector current we have

$$
\left(k_{1}-k_{2}\right)_{\nu} T_{\mu \nu}^{A V}=\int \frac{d^{4} k}{(2 \pi)^{4}} \operatorname{Tr}\left\{i \gamma_{\mu} \gamma_{5} \frac{1}{\left[\not k+\not k_{2}-m\right]}\right\}-\int \frac{d^{4} k}{(2 \pi)^{4}} \operatorname{Tr}\left\{i \gamma_{\mu} \gamma_{5} \frac{1}{\left[k+\not k+k_{1}-m\right]}\right\},
$$

where we immediately identify the one point axial functions which vanish identically and therefore $\left(k_{1}-k_{2}\right)_{\nu} T_{\mu \nu}^{A V}=0$. Doing the same for the axial current we have

$$
\begin{aligned}
\left(k_{1}-k_{2}\right)_{\mu} T_{\mu \nu}^{A V}= & -2 m i\left[T_{\nu}^{P V}\right]+\int \frac{d^{4} k}{(2 \pi)^{4}} \operatorname{Tr}\left\{i \gamma_{\nu} \gamma_{5} \frac{1}{\left[k+\not k_{2}-m\right]}\right\} \\
& -\int \frac{d^{4} k}{(2 \pi)^{4}} \operatorname{Tr}\left\{i \gamma_{\nu} \gamma_{5} \frac{1}{[k+\not k+m]}\right\},
\end{aligned}
$$

so that

$$
\left(k_{1}-k_{2}\right)_{\mu} T_{\mu \nu}^{A V}=-2 m i\left[T_{\nu}^{P V}\right],
$$

yielding the expected result for the Ward Identity in eq.(17). However the amplitude $T_{\nu}^{P V}$ is also identically zero given traces properties, which immediately implies with $\left(k_{1}-k_{2}\right)_{\mu} T_{\mu \nu}^{A V}=0$. If we take the four-divergence directly 
in eq.(83) it is easy to see that $\left(k_{1}-k_{2}\right)_{\nu} T_{\mu \nu}^{A V}=0$ and $\left(k_{1}-k_{2}\right)_{\mu} T_{\mu \nu}^{A V}=0$. We will analyze these results in the next section.

The case of the amplitude $T_{\mu \nu}^{V V}$ is analogous. If we first perform the contraction

$$
\begin{aligned}
\left(k_{1}-k_{2}\right)_{\mu} T_{\mu \nu}^{V V}= & \int \frac{d^{4} k}{(2 \pi)^{4}} \operatorname{Tr}\left\{\gamma_{\nu} \frac{1}{\left[\not k+\not k_{2}-m\right]}\right\} \\
& -\int \frac{d^{4} k}{(2 \pi)^{4}} \operatorname{Tr}\left\{\gamma_{\nu} \frac{1}{\left[\not k+\not k_{1}-m\right]}\right\}
\end{aligned}
$$

we again see that the result will depend on the $T_{\mu}^{V}$ one-point functions. Substituting now their expressions we get

$$
\begin{aligned}
\left(k_{1}-k_{2}\right)_{\mu} T_{\mu \nu}^{V V}=4\{ & \left(k_{1}-k_{2}\right)_{\alpha} \nabla_{\alpha \nu}+\left(k_{1 \alpha} k_{1 \beta} k_{1 \rho}-k_{2 \alpha} k_{2 \beta} k_{2 \rho}\right) \frac{\square_{\alpha \beta \rho \nu}}{3} \\
& \left.\quad\left(k_{1}^{2} k_{1 \rho}-k_{2}^{2} k_{2 \rho}\right) \frac{\triangle_{\rho \nu}}{3}-\left(k_{1 \nu} k_{1 \alpha} k_{1 \beta}-k_{2 \nu} k_{2 \alpha} k_{2 \beta}\right) \frac{2}{3} \triangle_{\alpha \beta}\right\}
\end{aligned}
$$

The conservation of the vector current demands, therefore that the r.h.s. be identically null. It is easy to check that similar results would have been obtained if we had taken the four-divergence directly on the final result for $T_{\mu \nu}^{V V}$, eq.(91). This shows that in order to satisfy the Ward Identities relative to the $T_{\mu \nu}^{V V}$ amplitude we need to require the same conditions as for the one-point function, eq.(123).

Last we turn to $T_{\mu \nu}^{A A}$. Associated to this Green's function we have two axial currents. This allows us to relate them to the amplitudes $T_{\mu}^{A P}$ and $T^{P P}$ by successive contractions with the external momentum. Thus, upon contracting with $\left(k_{1}-k_{2}\right)_{\mu}$ we get

$$
\begin{aligned}
\left(k_{1}-k_{2}\right)_{\mu} T_{\mu \nu}^{A A}= & -2 m i\left[T_{\nu}^{P A}\right]+\int \frac{d^{4} k}{(2 \pi)^{4}} \operatorname{Tr}\left\{\gamma_{\nu} \frac{1}{\left[\not k+\not k_{1}-m\right]}\right\} \\
& -\int \frac{d^{4} k}{(2 \pi)^{4}} \operatorname{Tr}\left\{\gamma_{\nu} \frac{1}{\left[\not k+\not k_{2}-m\right]}\right\},
\end{aligned}
$$

where the two last terms are again vector one-point functions and we get

$$
\begin{aligned}
\left(k_{1}-k_{2}\right)_{\mu} T_{\mu \nu}^{A A}=4\{ & \left(k_{2}-k_{1}\right)_{\alpha} \nabla_{\alpha \nu}+\left(k_{1 \alpha} k_{1 \beta} k_{1 \rho}-k_{2 \alpha} k_{2 \beta} k_{2 \rho}\right) \frac{\square_{\alpha \beta \rho \nu}}{3} \\
& \left.+\left(k_{1}^{2} k_{1 \rho}-k_{2}^{2} k_{2 \rho}\right) \frac{\triangle_{\rho \nu}}{3}+\left(k_{1 \nu} k_{1 \alpha} k_{1 \beta}-k_{2 \nu} k_{2 \alpha} k_{2 \beta}\right) \frac{2}{3} \triangle_{\alpha \beta}\right\}-2 m i T_{\nu}^{P A} .
\end{aligned}
$$

Similar results emerges if the contraction is performed with the expression in eq.(95). Therefore it is verified that the conditions under which the Ward Identities are satisfied are the same as the previous ones. In order to obtain the identity from eq.(20) it is enough to contract, once more, with the index $\nu$. However nothing new is produced.

\section{Three-Point Functions}

We next turn to the question of the verification of Ward Identities involving three-point functions. We will use only the first way pointed out in the introduction of this section, i.e., by relating the contracted functions with two-point functions, following Gertsein and Jackiw [1].

Let us consider the identity for the amplitude $T_{\lambda}^{V S S}$. Contracting the $\lambda$ vector Lorentz index with the external momentum of the vertex;

$$
\left(k_{3}-k_{2}\right)_{\lambda} T_{\lambda}^{V S S}=\int \frac{d^{4} k}{(2 \pi)^{4}} \operatorname{Tr}\left\{\hat{1} \frac{1}{\left[\not k+\not k_{1}-m\right]} \hat{1} \frac{1}{\left[\not k+\not k_{2}-m\right]}\left(\not k_{3}-\not k_{2}\right) \frac{1}{\left[\not k+\not k_{3}-m\right]}\right\}
$$

which can be written after the use of the identity

$$
\left(k_{3}-\not k_{2}\right)=\left(\not k+\not k_{3}-m\right)-\left(\not k+\not k_{2}-m\right)
$$

in the form: 


$$
\begin{aligned}
\left(k_{3}-k_{2}\right)_{\lambda} T_{\lambda}^{V S S}= & \int \frac{d^{4} k}{(2 \pi)^{4}} \operatorname{Tr}\left\{\hat{1} \frac{1}{\left[\not k+\not k_{1}-m\right]} \hat{1} \frac{1}{\left[\not k+\not k_{2}-m\right]}\right\} \\
& -\int \frac{d^{4} k}{(2 \pi)^{4}} \operatorname{Tr}\left\{\hat{1} \frac{1}{\left[\not k+\not k_{1}-m\right]} \hat{1} \frac{1}{\left[\not k+\not k_{3}-m\right]}\right\},
\end{aligned}
$$

where one can identify scalar two-point functions i.e.,

$$
\left(k_{3}-k_{2}\right)_{\lambda} T_{\lambda}^{V S S}\left(k_{1}, m ; k_{2}, m ; k_{3}, m\right)=T^{S S}\left(k_{1}, m ; k_{2}, m\right)-T^{S S}\left(k_{1}, m ; k_{3}, m\right) .
$$

Looking at eq.(70) we verify that the amplitude $T^{S S}$ possesses unambiguous terms. In the above expression this means terms which depends on differences $\left(k_{1}-k_{2}\right)^{2}$ and $\left(k_{1}-k_{3}\right)^{2}$ which are the external momenta $p^{\prime 2}$ and $p^{2}$, respectively. Explicitly the difference in eq.(145) can be written as

$$
\begin{aligned}
& \left(k_{3}-k_{2}\right)_{\lambda} T_{\lambda}^{V S S}\left(k_{1}, m ; k_{2}, m ; k_{3}, m\right)= \\
& \quad 2\left\{\left(4 m^{2}-p^{\prime 2}\right)\left[I_{l o g}\left(m^{2}\right)-\left(\frac{i}{(4 \pi)^{2}}\right) Z_{0}\left(m^{2}, m^{2}, p^{2} ; m^{2}\right)\right]\right. \\
& \left.\quad-\left(4 m^{2}-p^{2}\right)\left[I_{l o g}\left(m^{2}\right)-\left(\frac{i}{(4 \pi)^{2}}\right) Z_{0}\left(m^{2}, m^{2}, p^{2} ; m^{2}\right)\right]\right\} \\
& \quad+2\left(k_{2 \alpha} k_{2 \beta}-k_{3 \alpha} k_{3 \beta}\right) \triangle_{\alpha \beta} .
\end{aligned}
$$

Now we need to include the crossed channel. Redefining adequately the momenta of the external lines and operating in an analogous way we finally arrive at

$$
\begin{aligned}
& \left(l_{3}-l_{2}\right)_{\lambda} T_{\lambda}^{V S S}\left(l_{1}, m ; l_{2}, m ; l_{3}, m\right)= \\
& \quad 2\left\{\left(4 m^{2}-p^{2}\right)\left[I_{l o g}\left(m^{2}\right)-\left(\frac{i}{(4 \pi)^{2}}\right) Z_{0}\left(m^{2}, m^{2}, p^{2} ; m^{2}\right)\right]\right. \\
& \left.\quad-\left(4 m^{2}-p^{\prime 2}\right)\left[I_{l o g}\left(m^{2}\right)-\left(\frac{i}{(4 \pi)^{2}}\right) Z_{0}\left(m^{2}, m^{2}, p^{\prime 2} ; m^{2}\right)\right]\right\} \\
& \quad+2\left(l_{2 \alpha} l_{2 \beta}-l_{3 \alpha} l_{3 \beta}\right) \triangle_{\alpha \beta} .
\end{aligned}
$$

The sum of the contributions of the two channels yield the following expression for the searched Ward Identity

$$
q_{\lambda} T_{\lambda}^{V \rightarrow S S}=2\left(k_{2 \alpha} k_{2 \beta}-k_{3 \alpha} k_{3 \beta}\right) \triangle_{\alpha \beta}+2\left(l_{2 \alpha} l_{2 \beta}-l_{3 \alpha} l_{3 \beta}\right) \triangle_{\alpha \beta} .
$$

This result shows that the conservation of the vector current depends in this case on the difference $\triangle_{\alpha \beta}$.

For the amplitude $T_{\lambda}^{V \rightarrow P P}$ we find, proceeding in a complete similar way as the previous case,

$$
q_{\lambda} T_{\lambda}^{V \rightarrow P P}=2\left(k_{3 \alpha} k_{3 \beta}-k_{2 \alpha} k_{2 \beta}\right) \triangle_{\alpha \beta}+2\left(l_{3 \alpha} l_{3 \beta}-l_{2 \alpha} l_{2 \beta}\right) \triangle_{\alpha \beta} .
$$

Still with one Lorentz index we have the process $A \rightarrow S P$ for which we write first

$$
\left(k_{3}-k_{2}\right)_{\lambda} T_{\lambda}^{A S P}=\int \frac{d^{4} k}{(2 \pi)^{4}} \operatorname{Tr}\left\{\hat{1} \frac{1}{\left[\not k+\not k_{1}-m\right]} \gamma_{5} \frac{1}{\left[\not k+\not k_{2}-m\right]} i\left(k_{3}-\not k_{2}\right) \gamma_{5} \frac{1}{\left[\not k+\not k_{3}-m\right]}\right\} .
$$

Now, making use of a convenient identity

$$
\left(k_{2}-\not k_{3}\right) \gamma_{5}=\gamma_{5}\left[\not k+\not k_{3}-m\right]+\left[k+\not k_{2}-m\right] \gamma_{5}+2 m \gamma_{5}
$$

we can identify on the r.h.s. the two-point functions $T^{P P}$ and $T^{S S}$, besides $T^{P S P}$. Substituting the results for them with inclusion of the crossed channel, we have

$$
q_{\lambda} T_{\lambda}^{A \rightarrow S P}=-2 m i\left[T^{P \rightarrow S P}\right]-2 i\left(k_{3 \alpha} k_{3 \beta}-k_{2 \alpha} k_{2 \beta}\right) \triangle_{\alpha \beta}-2 i\left(l_{3 \alpha} l_{3 \beta}-l_{2 \alpha} l_{2 \beta}\right) \triangle_{\alpha \beta},
$$

where the symmetry violating terms are again associated with ambiguities.

The cases with two Lorentz indices are now in order. Making use of the two-point functions $T_{\mu}^{V S}$, for $T_{\mu \nu}^{S V V}$ we get for the two associated Ward Identities

$$
\begin{aligned}
p_{\mu} T_{\mu \nu}^{S \rightarrow V V} & =4 m\left(k_{3}-k_{1}\right)_{\alpha} \triangle_{\alpha \nu}+4 m\left(l_{1}-l_{2}\right)_{\alpha} \triangle_{\alpha \nu} \\
& =8 m p_{\alpha} \triangle_{\alpha \nu}
\end{aligned}
$$


and

$$
\begin{aligned}
p_{\nu}^{\prime} T_{\mu \nu}^{S \rightarrow V V} & =4 m\left(k_{1}-k_{2}\right)_{\alpha} \triangle_{\alpha \mu}+4 m\left(l_{3}-l_{1}\right)_{\alpha} \triangle_{\alpha \mu} \\
& =8 m p^{\prime}{ }_{\alpha} \triangle_{\alpha \mu} .
\end{aligned}
$$

In this results it is important to note an unambiguous character for the violating term. This means that the identity depends on the value of $\triangle_{\alpha \mu}$ and this is not associated with ambiguities here.

For the $S \rightarrow A A$ process, which has two related axial currents we get

$$
p_{\mu} T_{\mu \nu}^{S \rightarrow A A}=2 m i\left[T_{\nu}^{S \rightarrow P A}\right]-4 m\left(k_{2}+k_{3}\right)_{\alpha} \triangle_{\alpha \nu}-4 m\left(l_{2}+l_{3}\right)_{\alpha} \triangle_{\alpha \nu}
$$

and

$$
p_{\nu}^{\prime} T_{\mu \nu}^{S \rightarrow A A}=2 m i\left[T_{\mu}^{S \rightarrow A P}\right]-4 m\left(k_{3}+k_{2}\right)_{\alpha} \triangle_{\alpha \mu}-4 m\left(l_{3}+l_{2}\right)_{\alpha} \triangle_{\alpha \mu},
$$

which can be, in principle, violated by $\triangle_{\mu \nu}$ with ambiguous coefficients.

Let us now look to the process $V \rightarrow A P$. In the case of the vector current, the identity is expressed in terms of the two-point functions $T_{\mu}^{A P}$, which are unambiguous. Upon inclusion of the crossed channel we promptly obtain

$$
q_{\lambda} T_{\lambda \mu}^{V \rightarrow A P}=0 .
$$

On the other hand, the identity related to the axial current yields

$$
p_{\mu} T_{\lambda \mu}^{V \rightarrow A P}=2 m i\left[T_{\lambda}^{V \rightarrow P P}\right]-4 m\left(k_{2}+k_{3}\right)_{\alpha} \triangle_{\alpha \lambda}-4 m\left(l_{2}+l_{3}\right)_{\alpha} \triangle_{\alpha \lambda} .
$$

The two identities related to $T_{\mu \nu}^{P V V}$ are satisfied without restrictions since the two-point functions involved vanish identically. Thus

$$
\left(k_{3}-k_{1}\right)_{\mu} T_{\mu \nu}^{P V V}=0
$$

and

$$
\left(k_{1}-k_{2}\right)_{\nu} T_{\mu \nu}^{P V V}=0 .
$$

Also for the processes $V \rightarrow A S$ and $P \rightarrow A A$ both Ward Identities are satisfied by the same reason. Thus

$$
p_{\mu} T_{\mu \nu}^{S \rightarrow A V}=2 m i T_{\nu}^{S \rightarrow P V}
$$

and

$$
p_{\nu}^{\prime} T_{\mu \nu}^{S \rightarrow A V}=0
$$

Also

$$
\left\{\begin{array}{l}
p_{\mu} T_{\mu \nu}^{P \rightarrow A A}=2 m i T_{\nu}^{P \rightarrow P A} \\
p_{\nu}^{\prime} T_{\mu \nu}^{P \rightarrow A A}=2 m i T_{\mu}^{P \rightarrow A P}
\end{array}\right.
$$

We are now left with the amplitudes with three Lorentz indices. In the case of $T_{\lambda \mu \nu}^{V V V}$ the identities are expressed in terms of the two-point functions $T_{\mu \nu}^{V V}$ and we obtain

$$
\begin{aligned}
q_{\lambda} T_{\lambda \mu \nu}^{V \rightarrow V V}= & {\left[\left(k_{1}+k_{2}\right)_{\alpha}\left(k_{1}+k_{2}\right)_{\beta}-\left(k_{1}+k_{3}\right)_{\alpha}\left(k_{1}+k_{3}\right)_{\beta}\right]\left[\square_{\alpha \beta \mu \nu}-g_{\mu \alpha} \triangle_{\nu \beta}-g_{\nu \alpha} \triangle_{\mu \beta}-3 g_{\mu \nu} \triangle_{\alpha \beta}\right] } \\
& +\left[\left(l_{1}+l_{2}\right)_{\alpha}\left(l_{1}+l_{2}\right)_{\beta}-\left(l_{1}+l_{3}\right)_{\alpha}\left(l_{1}+l_{3}\right)_{\beta}\right]\left[\square_{\alpha \beta \mu \nu}-g_{\mu \alpha} \triangle_{\nu \beta}-g_{\nu \alpha} \triangle_{\mu \beta}-3 g_{\mu \nu} \triangle_{\alpha \beta}\right] \\
& +\frac{2}{3}\left[k_{1 \beta}\left(k_{3}-k_{2}\right)_{\alpha}-k_{1 \alpha}\left(k_{3}-k_{2}\right)_{\beta}+l_{1 \beta}\left(l_{3}-l_{2}\right)_{\alpha}-l_{1 \alpha}\left(l_{3}-l_{2}\right)_{\beta}\right]\left[\square_{\alpha \beta \mu \nu}+g_{\mu \alpha} \triangle_{\nu \beta}+g_{\nu \alpha} \triangle_{\mu \beta}\right] .
\end{aligned}
$$

Similar expression for $p_{\mu} T_{\lambda \mu \nu}^{V \rightarrow V V}$ and $p_{\nu}^{\prime} T_{\lambda \mu \nu}^{V \rightarrow V V}$ can be obtained. All conditions are expressed in terms of $\triangle_{\lambda \mu}$ and $\square_{\alpha \beta \nu \lambda}$.In the case of the $T_{\lambda \mu \nu}^{V A A}$ amplitude we have analogous results. For the vector current we find, after the standard operations,

$$
\left(k_{2}-k_{3}\right)_{\lambda} T_{\lambda \mu \nu}^{V \rightarrow A A}\left(k_{1}, m ; k_{2}, m ; k_{3}, m\right)=T_{\mu \nu}^{A A}\left(k_{1}, m ; k_{2}, m\right)-T_{\mu \nu}^{A A}\left(k_{1}, m ; k_{3}, m\right)
$$


and in consequence of the eq.(95), we may identify

$$
q_{\lambda} T_{\lambda \mu \nu}^{V \rightarrow A A}=-q_{\lambda} T_{\lambda \mu \nu}^{V \rightarrow V V}
$$

For the axial currents, in other hand, we set first

$$
\left(k_{3}-k_{1}\right)_{\mu} T_{\lambda \mu \nu}^{V \rightarrow A A}=2 m i T_{\lambda \nu}^{V \rightarrow P A}+T_{\lambda \nu}^{A A}\left(k_{1}, m ; k_{2}, m\right)-T_{\lambda \nu}^{V V}\left(k_{3}, m ; k_{2}, m\right)
$$

using now the results eq.(91) and eq.(95) and adding the contribution of the crossed channel, we set

$$
\begin{aligned}
p_{\mu} T_{\lambda \mu \nu}^{V \rightarrow A A}= & 2 m i T_{\lambda \nu}^{V \rightarrow P A} \\
& +\left[\left(l_{1}+l_{3}\right)_{\alpha}\left(l_{1}+l_{3}\right)_{\beta}-\left(l_{2}+l_{3}\right)_{\alpha}\left(l_{2}+l_{3}\right)_{\beta}\right]\left[\square_{\alpha \beta \lambda \nu}-g_{\lambda \beta} \triangle_{\nu \alpha}-g_{\lambda \alpha} \triangle_{\nu \beta}-3 g_{\lambda \nu} \triangle_{\alpha \beta}\right] \\
& -\left[\left(k_{1}+k_{2}\right)_{\alpha}\left(k_{1}+k_{2}\right)_{\beta}+\left(k_{2}+k_{3}\right)_{\alpha}\left(k_{2}+k_{3}\right)_{\beta}\right]\left[\square_{\alpha \beta \lambda \nu}-g_{\lambda \beta} \triangle_{\nu \alpha}-g_{\lambda \alpha} \triangle_{\nu \beta}-3 g_{\lambda \nu} \triangle_{\alpha \beta}\right] \\
& +\frac{2}{3}\left[k_{2 \alpha}\left(k_{1}+k_{3}\right)_{\beta}-k_{2 \beta}\left(k_{1}+k_{2}\right)_{\alpha}+l_{3 \alpha}\left(l_{1}-l_{2}\right)_{\beta}-l_{3 \beta}\left(l_{1}-l_{2}\right)_{\alpha}\right]\left[\square_{\alpha \beta \lambda \nu}+g_{\nu \alpha} \triangle_{\lambda \beta}+g_{\lambda \alpha} \triangle_{\nu \beta}\right] .
\end{aligned}
$$

A completely analogous condition can be obtained for the other axial current involved in $T_{\lambda \mu \nu}^{V \rightarrow A A}$.

Now we can finally investigate those associated to anomalous Ward Identity. First, for the $A V V$ amplitude, contracting with $\left(k_{3}-k_{2}\right)_{\lambda}$ before taking the traces, and using the identity

$$
\left(k_{2}-\not k_{3}\right) \gamma_{5}=\left(\not k+\not k_{2}-m\right) \gamma_{5}+\gamma_{5}\left(\not k+\not k_{3}-m\right)+2 m \gamma_{5}
$$

we get

$$
\begin{aligned}
\left(k_{3}-k_{2}\right)_{\lambda} T_{\lambda \mu \nu}^{A V V}= & -2 m i \int \frac{d^{4} k}{(2 \pi)^{4}} \operatorname{Tr}\left\{\gamma_{\mu} \frac{1}{\left[\not k+\not k_{1}-m\right]} \gamma_{\nu} \frac{1}{\left[\not k+\not k_{2}-m\right]} \gamma_{5} \frac{1}{\left[\not k+\not k_{3}-m\right]}\right\} \\
& -\int \frac{d^{4} k}{(2 \pi)^{4}} \operatorname{Tr}\left\{i \gamma_{\nu} \gamma_{5} \frac{1}{\left[\not k+\not k_{3}-m\right]} \gamma_{\mu} \frac{1}{\left[\not k+\not k_{1}-m\right]}\right\} \\
& +\int \frac{d^{4} k}{(2 \pi)^{4}} \operatorname{Tr}\left\{i \gamma_{\mu} \gamma_{5} \frac{1}{\left[\not k+\not k_{1}-m\right]} \gamma_{\nu} \frac{1}{\left[\not k+\not k_{2}-m\right]}\right\},
\end{aligned}
$$

which is the usual procedure [1], [4], [5], [6].

On the right hand side we can easily identify the three-point function $P V V$ and a pair of two-point functions $A V$. Substituting the appropriate value for two-point functions involved, eq.(83), and adding the corresponding crossed channel we obtain for the Ward Identity

$$
\begin{aligned}
q_{\lambda} T_{\lambda \mu \nu}^{A \rightarrow V V}= & -2 m i\left[T_{\mu \nu}^{P \rightarrow V V}\right] \\
& +2 \varepsilon_{\mu \nu \alpha \beta}\left[\left(k_{1}-k_{3}\right)_{\beta}\left(k_{1}+k_{3}\right)_{\xi}+\left(k_{2}-k_{1}\right)_{\beta}\left(k_{1}+k_{2}\right)_{\xi}\right] \triangle_{\xi \alpha} \\
& -2 \varepsilon_{\mu \nu \alpha \beta}\left[\left(l_{1}-l_{3}\right)_{\beta}\left(l_{1}+l_{3}\right)_{\xi}+\left(l_{2}-l_{1}\right)_{\beta}\left(l_{1}+l_{2}\right)_{\xi}\right] \triangle_{\xi \alpha}
\end{aligned}
$$

For the vector currents, first we contract with $\left(k_{3}-k_{1}\right)_{\mu}$ momentum, the direct channel, and make use of the identity

$$
\left(k_{3}-\not k_{1}\right)=\left(\not k+\not k_{3}-m\right)-\left(\not k+\not k_{1}-m\right)
$$

to obtain the relation

$$
\begin{aligned}
\left(k_{3}-k_{1}\right)_{\mu} T_{\lambda \mu \nu}^{A V V}= & \int \frac{d^{4} k}{(2 \pi)^{4}} \operatorname{Tr}\left\{i \gamma_{\lambda} \gamma_{5} \frac{1}{\left[\not k+\not k_{1}-m\right]} \gamma_{\nu} \frac{1}{\left[\not k+\not k_{2}-m\right]}\right\} \\
& -\int \frac{d^{4} k}{(2 \pi)^{4}} \operatorname{Tr}\left\{i \gamma_{\lambda} \gamma_{5} \frac{1}{\left[\not k+\not k_{3}-m\right]} \gamma_{\nu} \frac{1}{\left[\not k+\not k_{2}-m\right]}\right\}
\end{aligned}
$$

again the value assumed by $A V$ two-point function is crucial to define the value of the right hand side of the above equation. Using the results obtained in our calculation we get, after the addition of the crossed channel results,

$$
\begin{aligned}
p_{\mu} T_{\lambda \mu \nu}^{A \rightarrow V V}= & +2 \varepsilon_{\lambda \nu \alpha \beta}\left[\left(k_{2}-k_{1}\right)_{\beta}\left(k_{1}+k_{2}\right)_{\xi}+\left(k_{3}-k_{2}\right)_{\beta}\left(k_{2}+k_{3}\right)_{\xi}\right] \triangle_{\xi \alpha} \\
& +2 \varepsilon_{\lambda \nu \alpha \beta}\left[\left(l_{3}-l_{1}\right)_{\beta}\left(l_{1}+l_{3}\right)_{\xi}+\left(l_{2}-l_{3}\right)_{\beta}\left(l_{2}+l_{3}\right)_{\xi}\right] \triangle_{\xi \alpha} .
\end{aligned}
$$

In a completely analogous way, we arrive at the expression, for the other vector current involved; 


$$
\begin{aligned}
p_{\nu}^{\prime} T_{\lambda \mu \nu}^{A \rightarrow V V}= & +2 \varepsilon_{\lambda \mu \alpha \beta}\left[\left(k_{3}-k_{1}\right)_{\beta}\left(k_{1}+k_{3}\right)_{\xi}+\left(k_{2}-k_{3}\right)_{\beta}\left(k_{2}+k_{3}\right)_{\xi}\right] \triangle_{\xi \alpha} \\
& +2 \varepsilon_{\lambda \mu \alpha \beta}\left[\left(l_{2}-l_{1}\right)_{\beta}\left(l_{1}+l_{2}\right)_{\xi}+\left(l_{3}-l_{2}\right)_{\beta}\left(l_{2}+l_{3}\right)_{\xi}\right] \triangle_{\xi \alpha} .
\end{aligned}
$$

We repeat then the procedure for the three Ward Identities associated to $T_{\lambda \mu \nu}^{A A A}$. In this case, the contraction with the external momenta give rise again to two-point functions $T_{\mu \nu}^{A V}$ which, after some manipulation, yields

$$
\begin{aligned}
q_{\lambda} T_{\lambda \mu \nu}^{A \rightarrow A A}= & -2 m i T_{\mu \nu}^{P \rightarrow A A} \\
& +2 \varepsilon_{\mu \nu \alpha \beta}\left[\left(k_{3}-k_{1}\right)_{\beta}\left(k_{1}+k_{3}\right)_{\xi}+\left(k_{1}-k_{2}\right)_{\beta}\left(k_{1}+k_{2}\right)_{\xi}\right] \triangle_{\xi \alpha} \\
& +2 \varepsilon_{\mu \nu \alpha \beta}\left[\left(l_{3}-l_{1}\right)_{\beta}\left(l_{1}+l_{3}\right)_{\xi}+\left(l_{1}-l_{2}\right)_{\beta}\left(l_{1}+l_{2}\right)_{\xi}\right] \triangle_{\xi \alpha}
\end{aligned}
$$

and, in a completely analogous fashion,

$$
\begin{aligned}
p_{\mu} T_{\lambda \mu \nu}^{A \rightarrow A A}= & 2 m i T_{\lambda \nu}^{A \rightarrow P A} \\
& -2 \varepsilon_{\lambda \nu \alpha \beta}\left[\left(k_{1}-k_{2}\right)_{\beta}\left(k_{1}+k_{2}\right)_{\xi}+\left(k_{3}-k_{2}\right)_{\beta}\left(k_{2}+k_{3}\right)_{\xi}\right] \triangle_{\xi \alpha} \\
& +2 \varepsilon_{\lambda \nu \alpha \beta}\left[\left(l_{3}-l_{2}\right)_{\beta}\left(l_{1}+l_{3}\right)_{\xi}+\left(l_{1}-l_{3}\right)_{\beta}\left(l_{1}+l_{3}\right)_{\xi}\right] \triangle_{\xi \alpha} .
\end{aligned}
$$

Finally, for the last Ward Identity, we get:

$$
\begin{aligned}
p_{\nu}^{\prime} T_{\lambda \mu \nu}^{A \rightarrow A A}= & 2 m i T_{\lambda \mu}^{A \rightarrow A P} \\
& +2 \varepsilon_{\lambda \mu \alpha \beta}\left[\left(k_{1}-k_{3}\right)_{\beta}\left(k_{1}+k_{3}\right)_{\xi}+\left(k_{3}-k_{2}\right)_{\beta}\left(k_{2}+k_{3}\right)_{\xi}\right] \triangle_{\xi \alpha} \\
& +2 \varepsilon_{\lambda \mu \alpha \beta}\left[\left(l_{3}-l_{2}\right)_{\beta}\left(l_{2}+l_{3}\right)_{\xi}+\left(l_{1}-l_{2}\right)_{\beta}\left(l_{1}+l_{2}\right)_{\xi}\right] \triangle_{\xi \alpha} .
\end{aligned}
$$

Let us now proceed the final analysis of the preceding investigations.

\section{FINAL ANALYSIS}

For a free $1 / 2$ fermion, with equal masses, we have established the symmetry relations involving the divergent one, two and three-point functions. We next explicitly calculated, with arbitrary choices for the internal momenta of the loops, one and two-point functions and evaluated the potentially ambiguous part of the three-point functions. All the calculations have been performed from the point of view of a general calculational method in which only general assumptions are made in respect of an eventual regulator taken in an implicit way. No explicit calculations of a divergent integral are performed. In the result for the specific divergent integral, once no shifts are assumed, the results corresponding to other techniques are still maintained. To map these results into specific procedures all we need is to interpretate or evaluate the divergent terms according to the desired philosophy. In particular we have shown in the section $\mathrm{V}$ that the results corresponding to the traditional reference on this subject are still present in our results and a complete mapping is immediate. We are then at the position to analyze all the results looking at them and having in mind a searching for an universal interpretation of the possibilities of ambiguities and symmetry relation violations. In particular we are worried to answer the question: the justification of the anomaly phenomena can be consistently given in terms of ambiguities associated with (physically irrelevant) choices for the internal momenta (breaking of translational invariance)?. Our main argument to expect a negative answer to this question resides on the fact that if the anomalies are fundamental quantum phenomena therefore they must not be conditioned or justified in terms of divergences aspects, since, if the exact solutions were possible, then the description of the dynamics of the interacting particle would not involve divergences and, consequently, ambiguities. In other words, we believe that the ambiguities are typical consequences of the perturbative solution approach, as well as the infinities are, and a consistent interpretation of the perturbative amplitudes should leave no room for ambiguities like the renormalization process needs to eliminate all the divergences to furnish physical amplitudes. Any consistent treatment of the mathematical indefinitions associated to divergent integrals, from the physical point of view, needs to automatically eliminate the ambiguities. Let us now come from what we believe to what we obtain strictly from the point of view of the mathematical treatment.

We first note that in all calculated amplitudes the ambiguous dependence on the internal arbitrary momenta are always coefficients of only three combinations of divergent objects, with the same degree of divergence. They are

$$
\begin{aligned}
\square_{\alpha \beta \mu \nu}= & \int_{\Lambda} \frac{d^{4} k}{(2 \pi)^{4}} \frac{24 k_{\mu} k_{\nu} k_{\alpha} k_{\beta}}{\left(k^{2}-m^{2}\right)^{4}}-g_{\alpha \beta} \int_{\Lambda} \frac{d^{4} k}{(2 \pi)^{4}} \frac{4 k_{\mu} k_{\nu}}{\left[\left(k^{2}-m^{2}\right)^{3}\right]} \\
& -g_{\alpha \mu} \int_{\Lambda} \frac{d^{4} k}{(2 \pi)^{4}} \frac{4 k_{\beta} k_{\nu}}{\left[\left(k^{2}-m^{2}\right)^{3}\right]}-g_{\alpha \nu} \int_{\Lambda} \frac{d^{4} k}{(2 \pi)^{4}} \frac{4 k_{\beta} k_{\mu}}{\left[\left(k^{2}-m^{2}\right)^{3}\right]},
\end{aligned}
$$




$$
\begin{aligned}
& \nabla_{\mu \nu}=\int_{\Lambda} \frac{d^{4} k}{(2 \pi)^{4}} \frac{2 k_{\mu} k_{\nu}}{\left(k^{2}-m^{2}\right)^{2}}-\int_{\Lambda} \frac{d^{4} k}{(2 \pi)^{4}} \frac{g_{\mu \nu}}{\left(k^{2}-m^{2}\right)}, \\
& \triangle_{\mu \nu}=\int_{\Lambda} \frac{d^{4} k}{(2 \pi)^{4}} \frac{4 k_{\mu} k_{\nu}}{\left(k^{2}-m^{2}\right)^{3}}-\int_{\Lambda} \frac{d^{4} k}{(2 \pi)^{4}} \frac{g_{\mu \nu}}{\left(k^{2}-m^{2}\right)^{2}} .
\end{aligned}
$$

The second crucial observation is that all violating terms are also combinations of these objects but not all of them possess ambiguous coefficients. This means that even if shifts were allowed without restrictions the violating terms would be not completely removed. This statement is deeply related to the success of the Dimensional Regularization technique, which is well-known for producing amplitudes in a consistent way in respect ambiguities and symmetry relations. It is very easy to verify that in DR the objects $\square, \nabla$ and $\triangle$ vanish identically in such a way that all ambiguous and symmetry violating terms are automatically removed. Note that it is not sufficient to authorize shifts but it is necessary that the properties between divergent integrals, which we denominate consistency conditions, are assumed by the method because these aspects are not always associated. So, to obtain a perfect map from our result to those corresponding to DR calculations it is sufficient to eliminate $\square, \nabla$ and $\triangle$ and to evaluate the objects $I_{l o g}\left(m^{2}\right)$ and $I_{\text {quad }}\left(\mathrm{m}^{2}\right)$ from the point of view of that technique (taking the apropriate value for the traces of the $\gamma$ matrices $\operatorname{tr}\left(\gamma_{\mu} \gamma_{\nu}\right)=2^{\omega} g_{\mu \nu}$ and so on). The finiteness part is perfectly mapped one-by-one. These identifications are strictly possible for the amplitudes that can be obtained in $2 \omega$ dimensions ( $\omega=$ continuum and complex), i.e., to the cases where the number of the $\gamma_{5}$ involved matrix is not an odd quantity [25]. At this point it is not possible to run away from the observation: from our result it is possible to recover the DR results, where this technique could be applied, assuming $\square=\nabla=\triangle=0$. It is also possible to recover the surface term evaluations of the Gertsein-Jackiw approach in which case the result for $\square, \nabla$ and $\triangle$ cannot be zero. If we follow the way guided by DR philosophy, in what concerns the interpretation of the divergent objects, then we have obtained, from our general approach, all Ward Identity satisfied and all ambiguities are removed by the same condition that leads to this fact. Then the triangle anomaly phenomenon cannot be justified in a consistent way based on the divergent character of the involved amplitudes (ambiguities). If in the other hand we follow the Gertsein and Jackiw procedure then violations in Ward Identities of $A V V$ and $A A A$ amplitudes are obtained but a hard price must be paid because the ambiguities and violations would propagate to many other amplitudes which is a very undesirable feature of QFT in general, once renormalization become a very complicated scheme to be achieved in this fashion.

Another aspect involved in the Gertsein-Jackiw justification of the triangle anomaly phenomenon, which is quoted in many textbooks of QFT even nowadays [5] [6], is that the source of the violating term (anomaly) resides strictly on the value to be assumed by the $A V$ two-point function. This approach needs a nonzero value for this physical amplitude. This is a clearly inconsistent statement from many aspects of the QFT features. First, if the $A V$ amplitude does not vanish identically, it possesses two conserved currents: an axial-vector and a vector one, once we had:

$$
\begin{aligned}
& \left(k_{i}-k_{j}\right)_{\mu} T_{\mu \nu}^{A V}\left(k_{i}, m ; k_{j}, m\right)=0 \\
& \left(k_{i}-k_{j}\right)_{\nu} T_{\mu \nu}^{A V}\left(k_{i}, m ; k_{j}, m\right)=0,
\end{aligned}
$$

which is not consistent with the symmetry content of the model, in the sense that, to justify the violation of a symmetry relation we first need to violate another one, in such a way it is not possible to decide what is the fundamental symmetry breaking phenomenon. A second aspect is related to the unitarity. If the $A V$ amplitude does not vanish it needs to develop an imaginary part when the external momentum is $\left(k_{1}-k_{2}\right)^{2}=4 m^{2}$ (Cutkosky's rules). The value attributed to $A V$ amplitude by the Gertsein-Jackiw approach is not compatible with this QFT perturbative aspect. In addition, a CPT breaking may be introduced because the transition between a vector to axial-vector particle seems to be possible if the $A V$ two-point function becomes a nonzero quantity. Now we return again to specific analysis of our calculations.

In the present context, the analysis of the results, regarding the ambiguities and symmetry relations, resulted rather transparent, once we look all the conditions through the three consistency conditions above cited. Concerning this two aspects we can state what follows:

\section{- Ambiguities}

In all amplitudes the dependence arbitrary choices of internal momenta of loops appear simply as coefficients for the differences between divergent integrals, eqs.(178)-(180). Given this fact the conclusion is immediate: all the ambiguities will be eliminated if they are simultaneously zero. However, it is not surprising that ambiguities could be eliminated if shifts were allowed, i.e., provided we ignore the corresponding surface's terms, as we can see observing the conditions (123). Conversely, from this point of view, the conclusion can be looked as 4-D conditions to be satisfied by any regularization prescription which should have the consistency of DR whenever it applies. But this is not the whole story. 


\section{- Ward Identities}

In our investigations we have found several examples in which ambiguities and symmetry violations are intimately connected and have the same origin. However, there were also several instances in which Ward Identities could be violated by unambiguous terms with the same structure of the relations in the eqs.(178)-(180). This is the case, for example, of $T_{\mu \nu}^{V V}$ and $T_{\mu \nu}^{A A}$, where the difference $\nabla_{\alpha \beta}$ appears with an unambiguous coefficient. Also the three-point functions $T_{\mu \nu}^{S V V}$ and $T_{\mu \nu}^{S A A}$ involve the difference $\triangle_{\alpha \beta}$ as a condition for the fulfillment of the corresponding Ward Identities. The difference $\square_{\alpha \beta \mu \nu}$ would also appear with an unambiguous coefficient for the four-point function $T_{\mu \nu \lambda \rho}^{V V V V}$, among others, as it can be easily checked.

We could then invert the analysis starting precisely by these amplitudes and extracting the conclusion that, independently of ambiguities, the objects $\square_{\alpha \beta \mu \nu}, \nabla_{\mu \nu}$ and $\triangle_{\mu \nu}$ should be obtained as zero in a consistent treatment. We would then, a posteriori, verify that these conditions eliminate all sources of ambiguities.

At this point we reach an important and quite surprising result: Following the strategy of Gertsein and Jackiw in ref. [1] to study Ward Identities, which has historically been used to justify violations of symmetry relations, we found a set of conditions which allows all the WI to be satisfied. In this context the possibility of making use of ambiguities for any purpose is automatically eliminated. By imposing these referred conditions, the corresponding results of DR can be immediately mapped whenever it can be applied. Those corresponding Gertsein and Jackiw results, as we have shown in section $\mathrm{V}$, can be equally obtained from our results but not with the same interpretation for the objects $\square_{\alpha \beta \mu \nu}, \nabla_{\mu \nu}$ and $\triangle_{\mu \nu}$.

The situation is now the following: To establish or justify the existence of the anomaly phenomena, in the context of perturbative calculations, we need to use a specific prescription to evaluate some divergent integrals. The traditional one, based on surface terms, could be used, in principle, to treat all the amplitudes for any theories, but it is discarded nowadays where the DR can be applied and it is accepted only for the treatment of pseudo-amplitudes where the DR cannot be used due to its natural limitation. The two treatments lead to results that cannot be mapped one into another for places where both can be applied. If we are looking for an universal way to treat all divergent amplitudes in QFT, the above situation is unacceptable.

We arrive at two deeply different options: first, if we adopt the interpretation corresponding to the surface terms point of view we can get a picture for triangle anomalies that corresponds to the one of the Gertsein an Jackiw, but, in consequence, we will plague all physical amplitudes with ambiguities and consequently loose the translational invariance, the main one of the basic space-time symmetries implemented in the construction of QFT's. Second, if we adopt the DR interpretation for the objects $\square_{\alpha \beta \mu \nu}, \nabla_{\mu \nu}$ and $\triangle_{\mu \nu}$ we will have all symmetry relations satisfied, including those considered as anomalous.

This statement is an immediate consequence of our strategy in looking at perturbative calculations involving divergent amplitudes; the consistency conditions make immaterial an eventual choice for the value of undefined quantities, because in all places of occurrence they are multiplied by differences between divergent integrals of the same degree of divergence, that need to be identically zero by construction.

Once we have concluded that there is no chance of consistency in calculations involving divergent integrals without the imposition of the consistent conditions, a crucial question emerges: Do the combination of the treatment given to the divergent integral plus the strategy of Gertsein and Jackiw to verify Ward identities in three-point functions, lead to the conclusion that there are no anomalies in triangle diagrams? The answer is: not necessarily. The conclusion of our investigation, which at this point becomes transparent, is that this kind of analysis is not completely consistent, because we can find conditions that produce exactly the opposite conclusion of the initial intentions.

What is then the correct procedure to discuss this problem? Our expectation resides on the explicit calculations for the three-point functions and contracting them with the momenta only after the calculations are performed to generate Ward Identities. The correct violation values for symmetry relations (anomalies) required by phenomenological reasons like the neutral pion decay, need to emerge in a natural way, free from ambiguities related to the arbitrary choices of internal labels. The anomalous term needs to be associated with intrinsic properties of the involved three-point functions $T_{\lambda \mu \nu}^{A \rightarrow V V}$ and $T_{\mu \nu}^{P \rightarrow V V}$ and not with the value for a two-point function, $T_{\mu \nu}^{A V}$, which needs to be identically zero by many reasons. This is actually the essential point of the Sutherland-Veltman paradox [26] that states the impossibility to obtain simultaneously the three Ward Identities satisfied and the correct value for the amplitude $A V V$ in zero axial vertex momentum by a smooth limit. In the calculations here performed, and many others cited, these ingredients are not present and it is not reasonable to extract a conclusion about the triangle anomaly phenomenon.

In fact a work along these lines has been performed and a manuscript where all details involved in a completely general analytic calculation is in preparation [27]. Some results can be anticipated to give support to our final expectations and suppositions. After all the necessary calculations to give the most general analytic expression for $T_{\lambda \mu \nu}^{A \rightarrow V V}$ have been concluded, we can identify only one ambiguous term with the coefficient $\Delta_{\alpha \beta}$. The contraction with the external momenta gives us 


$$
\begin{aligned}
& \bullet p_{\mu} T_{\lambda \mu \nu}^{A \rightarrow V V}=\left(\frac{i}{4 \pi^{2}}\right) \varepsilon_{\nu \beta \lambda \xi} p_{\xi} p_{\beta}^{\prime} \\
& \bullet p_{\nu}^{\prime} T_{\lambda \mu \nu}^{A \rightarrow V V}=-\left(\frac{i}{4 \pi^{2}}\right) \varepsilon_{\mu \beta \lambda \xi} p_{\xi} p_{\beta}^{\prime} \\
& \bullet q_{\lambda} T_{\lambda \mu \nu}^{A \rightarrow V V}=-2 m i\left\{T_{\mu \nu}^{P \rightarrow V V}\right\}
\end{aligned}
$$

which shows that in adopting the value suggested by our investigation, $\Delta_{\alpha \beta}=0$, we do not spoil the violation. In addition we note that the value of the violating term is exactly that related to the $\lim _{q_{\lambda} \rightarrow 0} q^{\lambda} T_{\lambda \mu \nu}^{A V V}$ or that one we need for the phenomenological adjustment on the $A \rightarrow V V$ amplitude to allow, the neutral pion decay with a correct width. So, in the context of the Sutherland-Veltman paradox, if we choose an amplitude with a correct behavior on the $q_{\lambda} \rightarrow 0$ limit and simultaneously $U(1)$ gauge invariant for all values of the external momenta then an anomalous term should be included leading to the Ward identities

$$
\begin{aligned}
& \bullet p_{\nu}^{\prime}\left(T_{\lambda \mu \nu}^{A \rightarrow V V}\right)_{p h y}=0 \\
& \bullet p_{\mu}\left(T_{\lambda \mu \nu}^{A \rightarrow V V}\right)_{p h y}=0 \\
& \bullet q_{\lambda}\left(T_{\lambda \mu \nu}^{A \rightarrow V V}\right)_{p h y}=-2 m i\left\{T_{\mu \nu}^{P \rightarrow V V}\right\}-\left(\frac{i}{2 \pi^{2}}\right) \varepsilon_{\mu \nu \alpha \beta} p_{\alpha} p_{\beta}^{\prime} .
\end{aligned}
$$

Then, the $P V V$ amplitude in the right hand side of the eq. (188) can be identified as responsible for the neutral pion decay. It is important to emphasize the main aspect involved; the resulting amplitudes were derived without having recourse to ambiguities. The space-time homogeneity has been maintained. In addition the source of the anomalous term resides in strictly finite terms and then it is not necessarily associated to properties of divergent integrals and neither to the specific aspects of perturbative calculations. This is precisely what we should expect of a fundamental quantum phenomenon: to be still present in the eventual exact solutions consequently not revealed to us by the nature throughout the infinities which are nothing more than a consequence of our inability in solving the equations of motion of a QFT in an exactly way. Finally we call the attention that our results are in complete agreement with what we need to construct the renormalization of the Standard Model by anomaly cancellation.

[1] I.S. Gertsein and R. Jackiw, Phys. Rev. 181, 1955 (1969).

[2] G. 't Hooft and M. Veltman, Nucl. Phys. B44, 189 (1972); C.G. Bollini and J.J. Giambiagi, Phys. Lett. B40, 566 (1972); J.F. Ashmore, Nuovo Cimento Lett. 4, 289 (1972); G.M. Cicuta and E. Montaldi, Nuovo Cimento Lett. 4, 329 (1972).

[3] P.H. Frampton, Gauge Field Theories (Benjamin /Cummings, Menlo Park, California 1987, p. 206).

[4] S.L. Adler, Phys. Rev. 177, 2426 (1969); J.S. Bell and R. Jackiw, Nuovo Cim. 60A, 47 (1969); S.L. Adler, In Lectures on elementary particles and quantum field theory Proc.. 1970, Brandeis Summer Institute (ed. S. Deser et al.). MIT Press, Cambridge Massachussets; R. Jackiw, In Lectures on current algebra and its applications. Princeton University Press, Princeton, New Jersey (1972).

[5] M. Kaku, Quantum Field Theory (Oxford University Press) 1993, p. 414-419; T.P.Cheng and L.F. Li, Gauge Theory of Elementary Particle Physics (Oxford University Press, New York, 1984) p. 173-179; C. Itzykson and J.B. Zuber, Quantum Field Theory (McGraw-Hill, Inc, Singapore, 1980) p. 551-556; 3] p. 197-209; K. Huang, Quarks, Leptons Es Gauge Fields, (World Scientific) 1992 p. 231-237; S. Weinberg, The Quantum Theory of Fields, (Cambridge University Press) 1996 p. 370-383; 6] p.197-205.

[6] R.A. Bertlmann, Anomalies in Quantum Field Theory, (Oxford University Press) 1996.

[7] W.A. Bardeen, Phys. Rev. 184, 1848 (1969).

[8] O. Piguet and S.P. sorella, Algebraic Renormalization, (Spring-Verlag) 1995 p. 45-49; R. Jackiw and C. Rebbi, Phys. Rev. D14, 517 (1976); R. Jackiw and C. Rebbi, Phys. Rev. D16, 1052 (1977); N.K. Nielsen, H. Römer, Phys. Lett. B70, 445 (1977); H. Römer, Phys. Lett. B83, 172 (1979); H. Römer, Phys. Lett. B101, 55 (1981); K. Fujikawa, Phys. Rev. Lett. 42, 1195 (1979); K. Fujikawa, Phys. Rev. D21, 2848 (1980);

[9] R. Jackiw, Int. J. Mod. Phys. B14, 2011 (2000).

[10] Y. Nambu and J. Jona-Lasinio, Phys. Rev. 122, 345 (1961); A. Kocic, Phys. Rev. D33, 1785 (1986); D. Kohara, Phys. Lett. B229, 9 (1989); T. Hatsuda and T. Kunishiro, Phys. Rep. 247, 221 (1994); J. Bijnens, Phys. Rep. 265, 369 (1992).

[11] R.S. Willey, Phys. Rev. D48, 2877 (1993).

[12] T. Gherghetta, Phys. Rev. D50, 5985 (1994). 
[13] D. Colladay and V.A. Kostelecký, Phys. Rev. D55, 6760 (1997); D. Colladay and V.A. Kostelecký, Phys. Rev. D58, 116002 (1998); S. Coleman and S. Glashow, Phys. Rev. D59, 116008 (1999).

[14] R. Jackiw and V. Alan Kostelecký, Phys. Rev. Lett. 82, 3572 (1999).

[15] J.S. Chung and P. Oh, Phys. Rev. D60, 67702 (1999).

[16] W. F. Chen, Phys. Rev. D60, 85997 (1999).

[17] M. Pérez-Victoria, Phys. Rev. Lett. 83, 2518 (1999).

[18] M. Jaminon and E.R. Arriola, Phys. Lett. B433, 33 (1998); V. Dmitrasimovic, Eur. Phys.J. C14, 179 (2000).

[19] O.A. Battistel, PhD Thesis 1999, Universidade Federal de Minas Gerais, Brazil.

[20] O.A. Battistel and M.C. Nemes, Phys. Rev. D59, 055010 (1999).

[21] O.A. Battistel and G. Dallabona, Nucl. Phys. B610, 317 (2001); J. Phys. G27, L53-L60 (2001).

[22] A.P.B. Scarpelli, O.A. Battistel and M.C. Nemes, Braz.J. of Phys. 28, 161 (1998); S.R.Gobira, O.A.Battistel and M.C.Nemes, Braz. J. of Phys 30, 610 (2000).

[23] A. Brizola, O.A. Battistel, M. Sampaio and M.C. Nemes, Mod. Phys. Lett. A14, 1509 (1999).

[24] O.A. Battistel, A.L. Mota and M.C. Nemes, Mod. Phys. Lett. A13, 1557 (1998).

[25] J.C. Collins, Renormalization (Cambridge University Press, Cambridge, 1984); E. Manoukian, Renormalization (Academic Press), 1983); P. Ramond, Field Theory: A Modern Primer (Addison Wesley) 1990; S. Narison, Phys. Rep. 84 (1982) 263.

[26] D.G. Sutherland, Nucl. Phys. B2, 443 (1967); M. Veltman, Proc. R. Soc. A301, 107 (1967).

[27] O.A. Battistel, in preparation.

[28] G. Dallabona and O.A. Battistel, in preparation. 